MATHEMATICS OF COMPUTATION

Volume 74, Number 251, Pages 1231-1256

S 0025-5718(04)01706-5

Article electronically published on September 17, 2004

\title{
A DOMAIN DECOMPOSITION METHOD USING EFFICIENT INTERFACE-ACTING PRECONDITIONERS
}

\author{
SERGE KRÄUTLE
}

\begin{abstract}
The conjugate gradient boundary iteration (CGBI) is a domain decomposition method for symmetric elliptic problems on domains with large aspect ratio. High efficiency is reached by the construction of preconditioners that are acting only on the subdomain interfaces. The theoretical derivation of the method and some numerical results revealing a convergence rate of 0.04-0.1 per iteration step are given in this article. For the solution of the local subdomain problems, both finite element (FE) and spectral Chebyshev methods are considered.
\end{abstract}

\section{INTRODUCTION}

This article is concerned with a domain decomposition (DD) method for symmetric elliptic problems proposed by Borchers et al. 1] under the name conjugate gradient boundary iteration (CGBI). CGBI is based on a decomposition of the computational domain into nonoverlapping subdomains. In the present formulation, CGBI requires a domain decomposition without interior cross-points of the subdomain interfaces. Hence, it is best suited for domains with large aspect ratio, as they occur, e.g., for the computation of flows in a channel 3] (see Figure 11. In the theory of CGBI, the global problem is reformulated in terms of finding the correct natural boundary condition at the subdomain interfaces. The resulting dual problem for the boundary conditions is formulated as a minimization principle and is solved by preconditioned conjugate gradients (CG). Concerning the type of boundary conditions, CGBI resembles the finite element tearing and interconnecting (FETI) method by Farhat and Roux [8, 9, 10, 12, 17, 18, with the Neumann interface condition of CGBI corresponding to the Lagrangian multipliers of the FETI setting. One main difference between FETI and CGBI is that the CGBI preconditioner acts only on the subdomain interfaces instead of solving local problems on the subdomains. This is an advantage in terms of the computational costs, as the computational time for the CGBI interface preconditioner is negligible within a CG step, while the solution of another local problem on each subdomain, which is a widespread method of preconditioning for FETI [9, 12, 17, is almost as costly as the unpreconditioned CG step itself. Nevertheless, high convergence rates are achieved with the proposed boundary preconditioner. In fact, the convergence rate

Received by the editor October 18, 2003 and, in revised form, February 9, 2004.

2000 Mathematics Subject Classification. Primary 65N55; Secondary 65Y05, 65M70, 35J05.

Key words and phrases. Parallelization, domain decomposition, preconditioning, FETI.

This work was supported by the Deutsche Forschungsgemeinschaft (DFG) and the Centre National de la Recherche Scientifique (CNRS). 


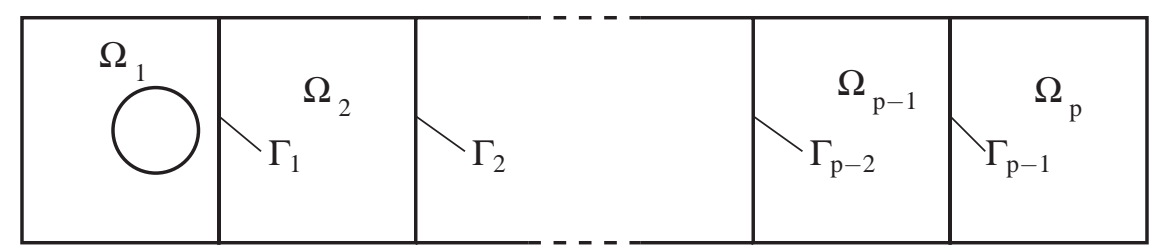

Figure 1. The decomposition of a computational domain as it occurs for the computation of the flow past an obstacle in a channel.

does not depend on the number of subdomains and the discretization parameter and is, at least for a model problem, smaller than for FETI.

DD methods like CGBI enable the coupling of different local solvers. The subdomain solvers considered in this article are finite element (FE) solvers and a Chebyshev spectral collocation solver. In [3], CGBI was used to couple an FE and the Chebyshev solver to compute the flow past obstacles in a channel. This coupling facilitates making use of the high efficiency of spectral methods on simple, rectangular parts of the computational domain with the flexibility of the FE method on more complicated parts.

The theory of CGBI is developed for the Poisson resolvent equation

$$
\mathcal{L} u:=\sigma u-\Delta u=f \quad \text { on } \Omega, \quad \Omega \subset \mathbb{R}^{n}, \quad \sigma \geq 0 .
$$

This equation is chosen for the following reason: (1.1) is the prototype of the equation that arises from a parabolic problem after temporal discretization $(\sigma \sim 1 / \Delta t$ with $\Delta t=$ timestep size). Furthermore, equation (1.1) derives from the temporal discretization of the Navier-Stokes equations if a pressure correction method (fractional step method) is used: After transformation to Lagrangian coordinates (practically, the computation of the foot points of the characteristics), a symmetric problem of type (1.1) with $\sigma \sim \operatorname{Re} / \Delta t, \operatorname{Re}=$ Reynolds number, has to be solved for each velocity component. In the last step, a Poisson equation (1.1) with $\sigma=0$ ) is solved for the pressure, and the velocity is projected [3]. Another method for handling parabolic equations, also using Neumann interface conditions, is described in 15 .

This article is structured as follows: In Section 2, the theoretical background of CGBI is presented. While the theory of the FETI method is based on a saddle point problem living on the union of all subdomain boundaries, in the center of the CGBI method there is an unconstrained minimization problem acting on the union of the subdomain interfaces. Another difference to the FETI approach is that, since we want to derive an estimate of the condition number independent 1 of the discretization parameter $h$, we focus on the nondiscretized problem. These estimates also hold (uniformly with respect to the discretization parameter) for the discretized problem using conforming linear finite elements. A result of this section is that the square root of a negative Laplace-Beltrami operator acting on the interfaces is an efficient preconditioner for the Poisson equation, leading to a condition number independent of the number of subdomains and the discretization parameter.

\footnotetext{
${ }^{1}$ Since we restrict ourselves to domain decompositions without inner cross-points, the typical polylogarithmic $h$-dependence of the condition number of Schur methods (cf. [6] and also [5, 12, 17, 18]) is suboptimal here.
} 
Section 3 deals with the question of how to discretize this interface preconditioner. Methods based on the fast Fourier transform and on sparse band matrices are proposed. Originally, these methods were developed for equidistant boundary meshes. Generalizations to uniform meshes and to a Chebyshev-Gauss-Lobatto boundary mesh are given.

In order to get an impression of the efficiency of CGBI, it is desirable to get a comparison of the condition number (the ratio of the largest and the smallest eigenvalue of the preconditioned operator) of CGBI to the preconditioned FETI method. If we restrict ourselves to a very simple computational domain with a simple domain decomposition, this computation of the condition number for the nondiscretized preconditioned operator can be done explicitly. As a result of Section 4 the CGBI condition number is only the square root of the condition number of FETI preconditioned with the well-known Dirichlet preconditioner [9].

In Section 5, numerical results for CGBI applied to the Poisson equation on 2 128 subdomains are presented. The performance of the preconditioners, both for linear finite element solvers and for Chebyshev spectral solvers, is demonstrated.

\section{Theory OF CGBI}

Let us consider a bounded domain $\Omega \subset \mathbb{R}^{n}$ with Lipschitz continuous boundary and the Poisson resolvent equation (1.1). For the sake of simplicity let us assume Dirichlet boundary conditions

$$
u=g \quad \text { on } \partial \Omega
$$

for the moment. For $f$ given in $H^{-1}(\Omega)$ which is defined as the dual space of $H_{0}^{1}(\Omega)$ we search for a weak solution $u \in H^{1}(\Omega)$ of (1.1)-(2.1).

2.1. The domain decomposition, definition of norms. For the solution of the given problem in parallel, let the domain $\Omega$ be decomposed into $p$ nonoverlapping subdomains $\Omega_{i}, i=1, \ldots, p$, in such a way that $\partial \Omega_{i}$ is Lipschitz continuous and $\Gamma_{i}:=\partial \Omega_{i} \cap \partial \Omega_{i+1} \neq \emptyset$ for $i=1, \ldots, p-1$ and $\partial \Omega_{i} \cap \partial \Omega_{j}=\emptyset$ else (see Figure 1).

We set $\Gamma=\bigcup_{i=1}^{p-1} \Gamma_{i}$. We denote by $\nu_{i}$ the outward unit normal on $\partial \Omega_{i}$. Obviously, $\nu_{i}=-\nu_{i+1}$ on $\Gamma_{i}$. For a function $u=\left(u_{1}, \ldots, u_{p}\right)$ defined on $\Omega, u_{i} \in H^{1}\left(\Omega_{i}\right)$, we define the jump $[u]$ on $\Gamma$ by $[u]=u_{i}-u_{i+1}$ on $\Gamma_{i}$. We denote by $H^{1 / 2}\left(\Gamma_{i}\right)$, $H_{00}^{1 / 2}\left(\Gamma_{i}\right)$ the usual fractional Sobolev spaces (trace spaces) (see, e.g., [14]) and by $H^{1 / 2}(\Gamma), H_{00}^{1 / 2}(\Gamma)$ the Cartesian products of the concerned spaces on $\Gamma_{i}$. The dual spaces of $H_{00}^{1 / 2}\left(\Gamma_{i}\right), H_{00}^{1 / 2}(\Gamma)$ are denoted by $H^{-1 / 2}\left(\Gamma_{i}\right), H^{-1 / 2}(\Gamma)$, and $\Delta_{\Gamma_{i}}$, $\Delta_{\Gamma}, \Delta_{\partial \Omega_{i}}$ are the Laplace-Beltrami operators whose domains are contained in $H_{0}^{1}\left(\Gamma_{i}\right), H_{0}^{1}(\Gamma), H^{1}\left(\partial \Omega_{i}\right)$, respectively. It is well known that for $u \in \bigotimes_{i=1}^{p} H^{1}\left(\Omega_{i}\right)$, $[u] \in H^{1 / 2}(\Gamma)$ holds, and for $u$ taken from the space

$$
H_{\partial \Omega}^{1}=\bigotimes_{i=1}^{p} H_{i, \partial \Omega}^{1},
$$

where

$$
H_{i, \partial \Omega}^{1}=\left\{u \in H^{1}\left(\Omega_{i}\right)|u|_{\partial \Omega \cap \partial \Omega_{i}}=0\right\},
$$

$[u] \in H_{00}^{1 / 2}(\Gamma)$ holds. 
Let $\sigma \in[0, \infty)$. On $H^{1}\left(\Omega_{i}\right), H_{00}^{1 / 2}\left(\Gamma_{i}\right), H^{1 / 2}\left(\partial \Omega_{i}\right)$ we define the norm:2

$$
\begin{aligned}
\|u\|_{\sigma, 1, \Omega_{i}}:= & \left\|(\sigma I-\Delta)^{1 / 2} u\right\|_{L^{2}\left(\Omega_{i}\right)} \sim\left(\sigma\|u\|_{L^{2}\left(\Omega_{i}\right)}^{2}+\|\nabla u\|_{L^{2}\left(\Omega_{i}\right)}^{2}\right)^{1 / 2} \\
\|\psi\|_{\sigma, \frac{1}{2}, \Gamma_{i}}:= & \left\|\left(\sigma I-\Delta_{\Gamma_{i}}\right)^{1 / 4} \psi\right\|_{L^{2}\left(\Gamma_{i}\right)} \\
& \sim\left(\sqrt{\sigma}\|\psi\|_{L^{2}\left(\Gamma_{i}\right)}^{2}+\left\|\left(-\Delta_{\Gamma_{i}}\right)^{1 / 4} \psi\right\|_{L^{2}\left(\Gamma_{i}\right)}^{2}\right)^{1 / 2} \\
\|\psi\|_{\sigma, \frac{1}{2}, \partial \Omega_{i}}:= & \left\|\left(\sigma I-\Delta_{\partial \Omega_{i}}\right)^{1 / 4} \psi\right\|_{L^{2}\left(\partial \Omega_{i}\right)} \\
& \sim\left(\sqrt{\sigma}\|\psi\|_{L^{2}\left(\partial \Omega_{i}\right)}^{2}+\left\|\left(-\Delta_{\partial \Omega_{i}}\right)^{1 / 4} \psi\right\|_{L^{2}\left(\partial \Omega_{i}\right)}^{2}\right)^{1 / 2}
\end{aligned}
$$

respectively. The equivalences ' $\sim$ ' can be derived by the spectral decomposition of the operators and are meant to hold uniformly with respect to $\sigma$. Using these norms, the well-known trace theorem can be generalized:

\section{Lemma 2.1.}

(a) The norm of the trace operator $T_{i}: H_{i, \partial \Omega}^{1} \rightarrow H_{00}^{1 / 2}\left(\Gamma_{i}\right)$, with respect to the norms (2.2), (2.3) is bounded uniformly with respect to $\sigma \in[0, \infty)$.

(b) There is a family of extension operators $P_{\sigma, i}: H_{00}^{1 / 2}\left(\Gamma_{i}\right) \rightarrow H^{1}\left(\Omega_{i}\right)$ that are continuous with respect to the norms (2.2), (2.3) and bounded uniformly with respect to $\sigma \in[0, \infty)$, such that $\left.\left(P_{\sigma, i} u\right)\right|_{\partial \Omega_{i} \backslash \Gamma_{i}}=0$.

Proof. See the Appendix.

The definitions (2.2), (2.3) lead in an obvious manner to the definition of the norms $\|\cdot\|_{\sigma, 1, \Omega},\|\cdot\|_{\sigma, 1 / 2, \Gamma}$ on the product spaces $H_{\partial \Omega}^{1}, H_{00}^{1 / 2}(\Gamma)$, resp., and their dual norms $\|\cdot\|_{\sigma,-1, \Omega},\|\cdot\|_{\sigma,-1 / 2, \Gamma}$.

2.2. The dual problem. For the statement of the CGBI algorithm we require a right-hand side $f=\left(f_{1}, \ldots, f_{p}\right)$ with $f_{i} \in H^{-1}\left(\Omega_{i}\right)$, which is only a slightly stronger assumption than that made on $f$ in the global problem (1.1)-(2.1).

CGBI for the problem (1.1)-(2.1) consists of a prestep and a main step. In the prestep, on each subdomain the boundary value problem

$$
\begin{aligned}
\mathcal{L} w_{i} & =f_{i} \text { in } \Omega_{i}, \\
w_{i} & =g \text { on } \partial \Omega_{i} \cap \partial \Omega, \\
\frac{\partial w_{i}}{\partial \nu_{i}} & =0 \text { on } \Gamma_{i-1} \cup \Gamma_{i}
\end{aligned}
$$

is solved $\left(\Gamma_{0}, \Gamma_{p}:=\emptyset\right)$, which can easily be done in parallel. If we decompose the solution $u$ of the global problem (1.1) as

$$
u_{i}=w_{i}+v_{i}
$$

on each $\Omega_{i}$, then the boundary value problem

$$
\begin{aligned}
\mathcal{L} v_{i} & =0 \text { in } \Omega_{i}, \\
v_{i} & =0 \text { on } \partial \Omega_{i} \cap \partial \Omega, \\
\frac{\partial v_{i}}{\partial \nu_{i}} & =-\varphi_{i-1} \text { on } \Gamma_{i-1}, \\
\frac{\partial v_{i}}{\partial \nu_{i}} & =\varphi_{i} \text { on } \Gamma_{i}
\end{aligned}
$$

\footnotetext{
${ }^{2}$ For $\sigma=0$, 2.2. and (2.4) are seminorms. On $H_{i, \partial \Omega}^{1}$, 2.4) is even for $\sigma=0$ a norm.
} 
(which will be denoted as the main step) follows for $v_{i}$ obviously. In (2.6), the boundary value function $\varphi=\left(\varphi_{1}, \ldots, \varphi_{p-1}\right)$ is a priori unknown. To make (2.6) meaningful, $\varphi$ has to be taken from the space $H^{-1 / 2}(\Gamma)$. Let us regard $v=$ $\left(v_{1}, \ldots, v_{p}\right)=v(\varphi)$ in (2.6) as a function of $\varphi$. By construction, the normal derivative of $v(\varphi)$ is continuous across the interfaces $\Gamma$. So $u(\varphi)=w+v(\varphi)$ is a solution of (1.1) if and only if the jump $[u(\varphi)]$ of $u(\varphi)$ at the interfaces is the zero function on $\Gamma$ (in the sense of traces). Hence, $u(\varphi)$ is the solution of (1.1)-(2.1) if $\varphi \in H^{-1 / 2}(\Gamma)$ fulfils the equation

$$
[v(\varphi)]=-[w]
$$

on $\Gamma$, or, in the weak formulation

$$
\langle\psi,[v(\varphi)]\rangle_{\Gamma}=-\langle\psi,[w]\rangle_{\Gamma} \quad \forall \psi \in H^{-1 / 2}(\Gamma) .
$$

$\langle\cdot, \cdot\rangle_{\Gamma}$ stands for the duality pairing between the spaces $H_{00}^{1 / 2}(\Gamma)$ and $H^{-1 / 2}(\Gamma)$.

Hence, we have reduced the problem (1.1) (2.1) to the dual problem (2.8) living only on the subdomain interfaces.

2.3. The bilinear form. In order to solve problem (2.8), we define the bilinear form

$$
b(\psi, \varphi)=\langle\psi,[v(\varphi)]\rangle_{\Gamma}
$$

on $H^{-1 / 2}(\Gamma) \times H^{-1 / 2}(\Gamma), v(\varphi)$ defined by (2.6). The corresponding operator is

$$
\mathcal{A}: \varphi \longmapsto[v(\varphi)], \quad H^{-1 / 2}(\Gamma) \longrightarrow H_{00}^{1 / 2}(\Gamma) .
$$

The solubility of (2.8) is guaranteed by the following theorem:

\section{Theorem 2.2.}

(i) The bilinear form $b$ is symmetric:

$$
b(\psi, \varphi)=b(\varphi, \psi)
$$

(ii) $b$ is continuous with respect to the $\sigma$-weighted norm of $H^{-1 / 2}(\Gamma)$ :

$$
b(\psi, \varphi) \leq c_{1}\|\psi\|_{\sigma,-\frac{1}{2}, \Gamma}\|\varphi\|_{\sigma,-\frac{1}{2}, \Gamma}
$$

(iii) $b$ is coercive:

$$
b(\varphi, \varphi) \geq c_{2}\|\varphi\|_{\sigma,-\frac{1}{2}, \Gamma}^{2}
$$

Hence,

$$
c_{2}\|\varphi\|_{\sigma,-\frac{1}{2}, \Gamma}^{2} \leq b(\varphi, \varphi) \leq c_{1}\|\varphi\|_{\sigma,-\frac{1}{2}, \Gamma}^{2}
$$

holds for all $\varphi \in H^{-1 / 2}(\Gamma)$. The constants $c_{1}, c_{2}>0$ depend on the norms of the local trace and prolongation operators on $\Omega_{i}, i=1, \ldots, p$. They can be chosen such that they are independent of the resolvent parameter $\sigma$, of global properties of $\Omega$ and of the number of subdomains $p$.

Before we prove Theorem 2.2, let us first state the consequences of the theorem:

Problem (2.8) has a unique solution, and due to the symmetry, there is a minimization principle

$$
J(\varphi):=\frac{1}{2} b(\varphi, \varphi)+\langle\varphi,[w]\rangle_{\Gamma} \longrightarrow \min \text { in } H^{-1 / 2}(\Gamma),
$$

and the classical CG algorithm can be used to compute the solution of 2.8)/(2.14). Let us remark that there is another well-known domain decomposition method, which was analysed by consideration of an underlying minimization principle, the 
Lions' method [16, 11]. It uses Robin-type interface conditions instead of our Neumann conditions, and its minimization principle acts on both the interfaces and the subdomains.

Furthermore, estimate (2.13) shows how to construct an efficient preconditioner for the operator $\mathcal{A}$ :

Let $\mathcal{C}$ be the Riesz isometry from the Hilbert space $H_{00}^{1 / 2}(\Gamma)$ with norm (2.3) to its dual $H^{-1 / 2}(\Gamma)$ :

$$
\mathcal{C}=\left(\sigma I-\Delta_{\Gamma}\right)^{1 / 2}
$$

Expressing the norm $\|\cdot\|_{\sigma,-\frac{1}{2}, \Gamma}$ in terms of $\mathcal{C},(2.13)$ is equivalent to

$$
c_{2}\left\|\mathcal{C}^{-1 / 2} \varphi\right\|_{L^{2}(\Gamma)}^{2} \leq b(\varphi, \varphi) \leq c_{1}\left\|\mathcal{C}^{-1 / 2} \varphi\right\|_{L^{2}(\Gamma)}^{2} \quad \forall \varphi \in H^{-1 / 2}(\Gamma) .
$$

Substituting $\psi=\mathcal{C}^{-1 / 2} \varphi$, we arrive at

$$
c_{2}\|\psi\|_{L^{2}(\Gamma)}^{2} \leq\left(\psi, \mathcal{C}^{1 / 2} \mathcal{A C}^{1 / 2} \psi\right)_{L^{2}(\Gamma)} \leq c_{1}\|\psi\|_{L^{2}(\Gamma)}^{2} \quad \forall \psi \in L^{2}(\Gamma)
$$

which is a main result of the CGBI theory. It reveals that $\mathcal{C}^{-1}$ is an efficient preconditioner for $\mathcal{A}$ : As the constants $c_{1}, c_{2}$ are independent of $\sigma$, the size of $\Omega$ and the number $p$ of subdomains, we can expect a condition number for the discretized problem (2.8), preconditioned with a discretized $\mathcal{C}^{-1}$, which is independent of $\sigma$, $|\Omega|, p$ and also of the discretization parameter, provided the discretization of $\mathcal{C}^{-1}$ is adequate. Therefore, the convergence rate of CGBI with respect to the norm $b(\varphi, \varphi)^{1 / 2}$ will be independent of these parameters. Note that this norm can be characterized by $b(\varphi, \varphi)^{1 / 2}=\|v(\varphi)\|_{\sigma, 1, \Omega}(\mathrm{cf}$. (2.17) $)$. By the Poincaré inequality in $H_{i, \partial \Omega}^{1},\|v(\varphi)\|_{1,1, \Omega} \leq c\|v(\varphi)\|_{\sigma, 1, \Omega}$ with a $c>0$ independent of $\sigma \in[0, \infty)$. So the convergence result carries over to $H^{1}$.

Since the preconditioned CG (PCG) algorithm requires the application of $\mathcal{C}$ to a vector, we will focus on a discretization of $\mathcal{C}$ instead of $\mathcal{C}^{-1}$, and we will frequently call $\mathcal{C}$ instead of $\mathcal{C}^{-1}$ our preconditioner. For possible discretizations of $\mathcal{C}$ see Section 3

If rectangular subdomains are used, the dependence of $c_{1}, c_{2}$ on the local trace and prolongation operators in Theorem 2.2 carries over to a dependence of $c_{1}, c_{2}$ on the aspect ratio of the subdomains. If a rather short channel is divided into many subdomains leading to a poor subdomain aspect ratio, $c_{1} / c_{2}$ becomes large. For quadratic subdomains or subdomains with length (in direction of the channel) bigger than height, $c_{1} / c_{2}$ is close to 1 (cf. Section 4.1).

Proof of Theorem 2.2. (i) Symmetry. It holds

$$
\begin{aligned}
b(\psi, \varphi) & =\sum_{i=1}^{p-1}\left\langle\psi_{i}, v_{i}(\varphi)-v_{i+1}(\varphi)\right\rangle_{\Gamma_{i}}=\sum_{i=1}^{p-1}\left\langle\psi_{i}, v_{i}(\varphi)\right\rangle_{\Gamma_{i}}+\left\langle-\psi_{i}, v_{i+1}(\varphi)\right\rangle_{\Gamma_{i}} \\
& =\sum_{i=1}^{p-1}\left\langle\frac{\partial v_{i}(\psi)}{\partial \nu_{i}}, v_{i}(\varphi)\right\rangle_{\Gamma_{i}}+\left\langle\frac{\partial v_{i+1}(\psi)}{\partial \nu_{i+1}}, v_{i+1}(\varphi)\right\rangle_{\Gamma_{i}} \\
& =\sum_{i=1}^{p-1}\left\langle\frac{\partial v_{i}(\psi)}{\partial \nu_{i}}, v_{i}(\varphi)\right\rangle_{\Gamma_{i}}+\sum_{i=2}^{p}\left\langle\frac{\partial v_{i}(\psi)}{\partial \nu_{i}}, v_{i}(\varphi)\right\rangle_{\Gamma_{i-1}} \\
& =\sum_{i=1}^{p}\left\langle\frac{\partial v_{i}(\psi)}{\partial \nu_{i}}, v_{i}(\varphi)\right\rangle_{\partial \Omega_{i} \backslash \partial \Omega}=\sum_{i=1}^{p}\left\langle\frac{\partial v_{i}(\psi)}{\partial \nu_{i}}, v_{i}(\varphi)\right\rangle_{\partial \Omega_{i}}
\end{aligned}
$$


Application of the divergence theorem on each subdomain $\Omega_{i}$ yields for $b$ the representation

$$
\begin{aligned}
b(\psi, \varphi) & =\sum_{i=1}^{p} \int_{\Omega_{i}}\left(v_{i}(\psi) \Delta v_{i}(\varphi)+\nabla v_{i}(\psi) \cdot \nabla v_{i}(\varphi)\right) d x \\
& =\sum_{i=1}^{p} \int_{\Omega_{i}}\left(\sigma v_{i}(\psi) v_{i}(\varphi)+\nabla v_{i}(\psi) \cdot \nabla v_{i}(\varphi)\right) d x .
\end{aligned}
$$

(ii) Continuity. From the representations (2.17) and (2.9) we get

$$
\begin{aligned}
& \|v(\varphi)\|_{\sigma, 1, \Omega}^{2}=b(\varphi, \varphi)=\sum_{i=1}^{p-1}\left\langle\varphi_{i}, v_{i}(\varphi)-v_{i+1}(\varphi)\right\rangle_{\Gamma_{i}} \\
& \leq \sum_{i=1}^{p-1}\left\|\varphi_{i}\right\|_{\sigma,-\frac{1}{2}, \Gamma_{i}}\left(\left\|v_{i}(\varphi)\right\|_{\sigma, \frac{1}{2}, \Gamma_{i}}+\left\|v_{i+1}(\varphi)\right\|_{\sigma, \frac{1}{2}, \Gamma_{i}}\right) .
\end{aligned}
$$

Using the generalized trace Lemma 2.1 the last line can be estimated by

$$
c_{T} \sum_{i=1}^{p-1}\left\|\varphi_{i}\right\|_{\sigma,-\frac{1}{2}, \Gamma_{i}}\left(\left\|v_{i}(\varphi)\right\|_{\sigma, 1, \Omega_{i}}+\left\|v_{i+1}(\varphi)\right\|_{\sigma, 1, \Omega_{i+1}}\right), \quad c_{T}>0 .
$$

(2.18) and the Cauchy-Schwarz inequality applied to (2.19) lead to

$$
\|v(\varphi)\|_{\sigma, 1, \Omega}^{2}=b(\varphi, \varphi) \leq 2 c_{T}\|\varphi\|_{\sigma,-\frac{1}{2}, \Gamma}\|v(\varphi)\|_{\sigma, 1, \Omega}
$$

A division yields

$$
\|v(\varphi)\|_{\sigma, 1, \Omega} \leq 2 c_{T}\|\varphi\|_{\sigma,-\frac{1}{2}, \Gamma} .
$$

Following (2.18) $-(2.20)$ with $b(\varphi, \varphi)$ replaced by $b(\psi, \varphi)$, we get similarly

$$
b(\psi, \varphi) \leq 2 c_{T}\|\psi\|_{\sigma,-\frac{1}{2}, \Gamma}\|v(\varphi)\|_{\sigma, 1, \Omega} \leq 4 c_{T}^{2}\|\psi\|_{\sigma,-\frac{1}{2}, \Gamma}\|\varphi\|_{\sigma,-\frac{1}{2}, \Gamma}
$$

where we used (2.21) in the last step.

(iii) Coercivity. By definition,

$$
\|\varphi\|_{\sigma,-\frac{1}{2}, \Gamma}=\sup _{\substack{\psi \in H_{00}^{1 / 2}(\Gamma) \\\|\psi\|_{\sigma, \frac{1}{2}, \Gamma}=1}} \sum_{i=1}^{p-1}\left\langle\varphi_{i}, \psi_{i}\right\rangle_{\Gamma_{i}}
$$

holds. Due to Lemma 2.1 every $\psi_{i} \in H_{00}^{1 / 2}\left(\Gamma_{i}\right)$ can be extended continuously to a $H^{1}\left(\Omega_{i}\right)$ function $P_{\sigma, i} \psi_{i}$ such that $\left.\left(P_{\sigma, 1} \psi_{i}\right)\right|_{\partial \Omega_{i} \backslash \Gamma_{i}}=0$, and the continuity of $P_{\sigma, i}$ is uniform with respect to $\sigma$. Hence, $P_{\sigma} \psi=\left(P_{\sigma, 1} \psi_{1}, \ldots, P_{\sigma, p-1} \psi_{p-1}, 0\right)$ depends continuously on $\psi$ :

$$
\left\|P_{\sigma} \psi\right\|_{\sigma, 1, \Omega} \leq c_{P}\|\psi\|_{\sigma, \frac{1}{2}, \Gamma}
$$


$c_{P}>0$ independent of $\sigma$. For the duality pairing in (2.22) we get

$$
\begin{aligned}
& \left\langle\varphi_{i}, \psi_{i}\right\rangle_{\Gamma_{i}}=\left\langle\frac{\partial}{\partial \nu_{i}} v_{i}(\varphi),\left.\left(P_{\sigma, i} \psi_{i}\right)\right|_{\partial \Omega_{i}}\right\rangle_{\partial \Omega_{i}} \\
& =\int_{\Omega_{i}}\left(\sigma v_{i}(\varphi) P_{\sigma, i} \psi_{i}+\nabla v_{i}(\varphi) \cdot \nabla P_{\sigma, i} \psi_{i}\right) d x \\
& \leq\left\|v_{i}(\varphi)\right\|_{\sigma, 1, \Omega_{i}}\left\|P_{\sigma, i} \psi_{i}\right\|_{\sigma, 1, \Omega_{i}} .
\end{aligned}
$$

Summation over $i$, the Cauchy-Schwarz inequality, and (2.23) yield

$$
\langle\varphi, \psi\rangle_{\Gamma} \leq c_{P}\|v(\varphi)\|_{\sigma, 1, \Omega}\|\psi\|_{\sigma, \frac{1}{2}, \Gamma} .
$$

Hence, (2.22) evaluates to

$$
\|\varphi\|_{\sigma,-\frac{1}{2}, \Gamma} \leq c_{P}\|v(\varphi)\|_{\sigma, 1, \Omega}=c_{P} b(\varphi, \varphi)^{1 / 2} .
$$

2.4. Generalization to the case of "floating subdomains". Let us consider (1.1) with

$$
\sigma=0
$$

in this section.

If Neumann boundary conditions

$$
\frac{\partial u}{\partial \nu}=g
$$

are imposed on a part $\partial \Omega_{N m}$ of $\partial \Omega$ and Dirichlet conditions (2.1) on $\partial \Omega_{D i r}=$ $\partial \Omega \backslash \partial \Omega_{N m}, 3$ the problem of the so-called floating subdomains may occur. These are subdomains $\Omega_{i}$ with pure Neumann boundary, i.e., $\partial \Omega_{i} \cap \partial \Omega_{D i r}=\emptyset$. If we would merely adapt the boundary conditions on $\partial \Omega_{i} \cap \partial \Omega_{N m}$ in the prestep (2.5) and in the main problem (2.6), those problems would be ill posed on the floating subdomains. Therefore modifications to these subproblems are necessary to ensure the existence and uniqueness of the local solutions. To keep the notation simple, let us assume that $\Gamma_{D i r} \subset \partial \Omega_{p}$, i.e., all $\Omega_{1}, \ldots, \Omega_{p-1}$ are floating.

In the prestep (2.5), the interface boundary condition is modified to

$$
\frac{\partial w_{i}}{\partial \nu_{i}}=c_{i} \text { on } \Gamma_{i}, \quad \frac{\partial w_{i}}{\partial \nu_{i}}=-c_{i-1} \text { on } \Gamma_{i-1}, \quad i=1, \ldots, p,
$$

where the $c_{1}, \ldots, c_{p-1} \in \mathbb{R}$ are constants chosen such that the compatibility conditions

$$
\left\langle f_{i}, 1\right\rangle_{\Omega_{i}}+\langle g, 1\rangle_{\partial \Omega_{i} \cap \partial \Omega}+c_{i}\left|\Gamma_{i}\right|-c_{i-1}\left|\Gamma_{i-1}\right|=0, \quad i=1, \ldots, p-1,
$$

$c_{0}:=0$, are met. Equation (2.25) serves as a recurrency equation for the $c_{i}$. So far, the local solutions $w_{i}$ are only defined up to a constant. The uniqueness of the $w_{i}$ is guaranteed by imposing the constraint $\int_{\Gamma_{i}}[w]=0, i=1, \ldots, p-1$, which is reached by adding a suitable constant to each $w_{i}, i=p-1, \ldots, 1$.

Concerning the main problem (2.6) and the corresponding bilinear form $b$, we replace the function spaces of the Dirichlet case for the interface conditions $\varphi$, for the local solutions $u(\varphi)$ and for the jumps $[u(\varphi)]$.

\footnotetext{
${ }^{3}$ Such problems occur for the pressure in the computation of a Navier-Stokes flow by a pressure correction scheme.
} 
The assumption $\varphi \in H_{N m}^{-1 / 2}(\Gamma)$, where $H_{N m}^{-1 / 2}(\Gamma)$ is the dual space of

$$
H_{N m}^{1 / 2}(\Gamma)=\left\{\psi \in H^{1 / 2}(\Gamma) \mid \int_{\Gamma_{i}} \psi_{i} d o=0, i=1, \ldots, p-1\right\},
$$

ensures the solubility of the Neumann problem corresponding to (2.6). As in the prestep, the uniqueness of the solution $v(\varphi)$ is implied by imposing $\int_{\Gamma_{i}}[v(\varphi)]=0$, $i=1, \ldots, p-1$. These constraints for $v(\varphi)$ and for $w$ are chosen such that the jumps $[v(\varphi)],[w]$ are in the space $H_{N m}^{1 / 2}(\Gamma)$.

The prestep and the main problem now read as follows:

$$
\begin{aligned}
\mathcal{L} w_{i} & =f_{i} \text { in } \Omega_{i} \\
w_{i} & =g \text { on } \partial \Omega_{i} \cap \partial \Omega_{D i r}, \\
\frac{\partial w_{i}}{\partial \nu_{i}} & =g \text { on } \partial \Omega_{i} \cap \partial \Omega_{N m}, \\
\frac{\partial w_{i}}{\partial \nu_{i}} & =-c_{i-1} \text { on } \Gamma_{i-1} \quad(\text { if } i>1), \\
\frac{\partial w_{i}}{\partial \nu_{i}} & =c_{i} \text { on } \Gamma_{i} \quad(\text { if } i<p), \\
\int_{\Gamma_{i}} w_{i} d o & =\int_{\Gamma_{i}} w_{i+1} d o \quad(\text { if } i<p)
\end{aligned}
$$

for $i=1, \ldots, p$, where $c_{0}=0, c_{i}=\frac{1}{\left|\Gamma_{i}\right|}\left(c_{i-1}\left|\Gamma_{i-1}\right|-\left\langle f_{i}, 1\right\rangle_{\Omega_{i}}-\langle g, 1\rangle_{\partial \Omega_{i} \cap \partial \Omega}\right)$ and

$$
\begin{aligned}
\mathcal{L} v_{i} & =0 \text { in } \Omega_{i} \\
v_{i} & =0 \text { on } \partial \Omega_{i} \cap \partial \Omega_{D i r}, \\
\frac{\partial v_{i}}{\partial \nu_{i}} & =0 \text { on } \partial \Omega_{i} \cap \partial \Omega_{N m}, \\
\frac{\partial v_{i}}{\partial \nu_{i}} & =-\varphi_{i-1} \text { on } \Gamma_{i-1} \quad(\text { if } i>1), \\
\frac{\partial v_{i}}{\partial \nu_{i}} & =\varphi_{i} \text { on } \Gamma_{i} \quad(\text { if } i<p), \\
\int_{\Gamma_{i}} v_{i} d o & =\int_{\Gamma_{i}} v_{i+1} d o \quad(\text { if } i<p)
\end{aligned}
$$

for $i=1, \ldots, p$. Note that the computation and addition of constants in (2.26)(2.27) play the role of the coarse system (the projection) in the FETI theory [9. So this step becomes rather trivial for the CGBI substructuring of a channel.

For the new definition of $b: H_{N m}^{-1 / 2}(\Gamma) \times: H_{N m}^{-1 / 2}(\Gamma) \rightarrow \mathbb{R}$ including the new definition of $v(\varphi)$ by (2.27), Theorem 2.2 also holds in the presence of floating subdomains.

In deviation from the proof of the coercivity in the Dirichlet case, one constructs a continuous extension operator from $H^{1 / 2}\left(\Gamma_{i}\right)$ to $\left\{u \in H^{1}\left(\Omega_{i}\right)|u|_{\Gamma_{i-1}}=0\right\}$. Note that in the space $\left\{v \in \bigotimes_{i=1}^{p} H^{1}\left(\Omega_{i}\right)\left|\int_{\Gamma_{i}} v_{i}=\int_{\Gamma_{i}} v_{i+1}, v_{n}\right|_{\partial \Omega_{D i r}}=0\right\}$ (containing the solutions $v(\varphi)$ of (2.27) ) a Poincaré inequality holds, as those integral mean values of the traces depend continuously on $v_{i}, v_{i+1}$ (e.g., [13, Sec. 2.4.3]). Hence, the seminorm $\left(\sum_{i}\left\|\nabla v_{i}\right\|_{L^{2}\left(\Omega_{i}\right)}^{2}\right)^{1 / 2}$, for which we derive the convergence result, is truly a norm on that space. 
The derivation of the preconditioner $\mathcal{C}$ for the Dirichlet case in Section 2.3 carries over to the preconditioner

$$
\mathcal{C}_{N m}=\left(\sigma I-\Delta_{N m}\right)^{1 / 2}
$$

or $\mathcal{C}_{N m}=\left(-\Delta_{N m}\right)^{1 / 2}$ (since $\sigma=0$ ) for the Neumann case, where $-\Delta_{N m}$ is the Laplace-Beltrami operator with homogeneous Neumann boundary conditions whose domain is contained in $H^{1}(\Gamma)$.

\section{The CONSTRUCTION OF PRECONDITIONERS}

Let us restrict ourselves to the case $\Omega \subset \mathbb{R}^{2}$. The interfaces are Lipschitz curves which can be parametrized by their arc length. To keep the notation simple, let us identify each interface with the interval $[0, L]$.

3.1. Preconditioners on equidistant boundary mesh. In Sections 2.3 and 2.4 we motivated the use of the preconditioner (2.15) in the case of Dirichlet conditions at $\partial \Omega \cap \Gamma_{i}$ and (2.28) in the case of $\sigma=0$ and Neumann conditions at $\partial \Omega \cap \Gamma_{i}$. For the discretization of the operators

$$
\mathcal{C}=\left(\sigma I-\Delta_{\Gamma_{i}}\right)^{1 / 2}, \quad \mathcal{C}_{N m}=\left(\sigma I-\Delta_{N m}\right)^{1 / 2}
$$

on each $\Gamma_{i}$ we propose the following two approaches:

3.1.1. The spectral preconditioner. Obviously, $s_{k}(x)=\sin \frac{k \pi x}{L}, k \in \mathbb{N}$, is an orthogonal basis in $H_{00}^{1 / 2}([0, L])$ and $c_{k}(x)=\cos \frac{k \pi x}{L}, k \in \mathbb{N}$, is an orthogonal basis in $H_{N m}^{1 / 2}([0, L])$. A given boundary value $\psi$ can be decomposed with respect to this basis. In the Fourier space, the action of $\mathcal{C}\left(\mathcal{C}_{N m}\right)$ is easily described:

$$
\begin{gathered}
\mathcal{C}: \psi=\sum_{k \geq 1} \alpha_{k} s_{k} \longmapsto \sum_{k \geq 1} \alpha_{k} \sqrt{\sigma+\left(\frac{k \pi}{L}\right)^{2}} s_{k}, \\
\mathcal{C}_{N m}: \psi=\sum_{k \geq 1} \alpha_{k} c_{k} \longmapsto \sum_{k \geq 1} \alpha_{k} \sqrt{\sigma+\left(\frac{k \pi}{L}\right)^{2}} c_{k} .
\end{gathered}
$$

As a discretization $\mathcal{C}^{h}\left(\mathcal{C}_{N m}^{h}\right)$, we use the following algorithm:

(1) For $\psi$ given on equidistantly spaced mesh points $x_{0}, \ldots, x_{N}$ on $[0, L]=\Gamma_{i}$, compute the discrete sine or cosine coefficients $\alpha_{k}$, respectively, by the fast Fourier transform (FFT).

(2) Perform $\alpha_{k} \mapsto \sqrt{\sigma+(k \pi / L)^{2}} \alpha_{k}$.

(3) Get the discrete values of $\mathcal{C}^{h} \psi\left(\mathcal{C}_{N m}^{h} \psi\right)$ by application of $F F T^{-1}$.

This preconditioner only takes $O(N \log N)$ operations. Assuming that there are about $N^{2}$ mesh points within $\Omega_{i}$ (which would be typical for a quadratic subdomain), the computational costs of the preconditioner are clearly negligible compared to the costs of the local solver (which are at least $O\left(N^{2}\right)$ operations). This is the main difference between CGBI and FETI: For the FETI preconditioning, another local problem on each subdomain has to be solved [9] which approximately doubles the computational costs per CG iteration step. 
3.1.2. Matrix preconditioners. Another approach is to approximate the above mapping by a (sparse) band matrix. Let us focus on the case $\sigma=0$; the generalization to $\sigma>0$ is obvious.

Clearly, the discrete spectral preconditioner $\mathcal{C}^{h}\left(\mathcal{C}_{N m}^{h}\right)$ proposed in Section 3.1.1 has the eigenvalues $\lambda_{k}=k \pi / L, k=1, \ldots, N-1(k=1, \ldots, N$, resp. $)$ and the eigenvectors $v_{k}=\left(v_{k, i}\right)_{i=0, \ldots, N}, v_{k, i}=\sin \frac{k i \pi}{N}\left(v_{k, i}=\cos \frac{k i \pi}{N}\right.$, resp.). One can prove [13] that the symmetric band matrix $\mathcal{C}_{M}$ with entries

$$
a_{j}= \begin{cases}\pi+1-2^{-\left\lfloor\log _{2} N\right\rfloor}, & j=0, \\ -\frac{\pi}{2}, & j=1, \\ -\frac{1}{2 j}, & j=2,4,8,16, \ldots, \\ 0, & \text { elsewhere }\end{cases}
$$

on the $j^{\text {th }}$ diagonal (after consideration of the boundary condition $\left.{ }^{4}\right)$ is spectrally equivalent in the sense that for the condition numbers $\kappa$

$$
c_{1} \kappa\left(\mathcal{C}^{h}\right) \leq \kappa\left(\mathcal{C}_{M}\right) \leq c_{2} \kappa\left(\mathcal{C}^{h}\right)
$$

holds with constants $c_{1}, c_{2}>0$ independent of $N$. As $\mathcal{C}_{M}$ contains only about $\log _{2} N$ nonzero bands, the application of $\mathcal{C}_{M}$ as a preconditioner again takes $O(N \log N)$ operations.

A discretization even simpler than (3.3) is given by the symmetric tridiagonal matrix $\mathcal{C}_{\text {tri }}$ with entries $a_{0}=1 / 2+1 / N, a_{1}=-1 / 4$. However, this discretization is not spectrally equivalent to $\mathcal{C}^{h}$ with constants independent of $N$ [13].

3.2. Preconditioners on uniform boundary meshes. Both the spectral and the matrix preconditioner in the present form make use of the fact that the discrete values of $\psi_{h}$ on $\Gamma_{i}=[0, L]$ are equidistantly spaced. This section deals with the case when the meshes on the subdomains $\Omega_{i}, \Omega_{i+1}$ coincide on $\Gamma_{i}$ but are not equidistant 5 It is not essential to consider the full $\sigma$-dependent operator, since the $\sigma$-dependent part can be split off by the equivalence

$$
\left(\sigma I-\Delta_{\Gamma_{i}}\right)^{1 / 2} \sim \sqrt{\sigma} I+\left(-\Delta_{\Gamma_{i}}\right)^{1 / 2}
$$

For $h>0$ let $\mathcal{M}_{h}$ be a mesh $0=x_{0}<x_{1}<\cdots<x_{N}=L$ on $[0, L]$. For each $\mathcal{M}_{h}$ we define $h_{\text {max }}=\max \left\{x_{i+1}-x_{i}\right\}, h_{\text {min }}=\min \left\{x_{i+1}-x_{i}\right\}, h_{\text {mean }}=L / N$. We define a piecewise linear mesh distribution function $w_{h}:[0, L] \rightarrow[0, L]$ with $w_{h}(i L / N)=x_{i}$. Hence, $w_{h}$ maps an equidistant mesh onto the given mesh, and the Lipschitz property

$$
\frac{h_{\text {min }}}{h_{\text {mean }}} \leq \frac{w_{h}(x)-w_{h}(y)}{x-y} \leq \frac{h_{\text {max }}}{h_{\text {mean }}}
$$

holds.

Lemma 3.1. (i) Let the mesh be uniform, i.e.,

$$
h_{\min } \geq d h_{\max }
$$

\footnotetext{
${ }^{4}(3.3)$ reflects the situation for periodic boundary conditions. For Dirichlet or Neumann conditions, some matrix entries are modified in such a way that $\mathcal{C}_{M}$ still has the same eigenvectors as $\mathcal{C}^{h}\left(\mathcal{C}_{N m}^{h}\right)$.

${ }^{5}$ If the meshes of $\Omega_{i}$ and $\Omega_{i+1}$ do not coincide on $\Gamma_{i}$, an interpolation of the discrete boundary values onto a common boundary mesh is done.
} 
with $d>0$ independent of $h$ for all meshes under consideration. Then, for any $\psi_{h} \in H^{1 / 2}([0, L])$, the function $\tilde{\psi}_{h}:[0, L] \rightarrow \mathbb{R}$ defined by $\tilde{\psi}_{h}=\psi_{h} \circ w_{h}$ is in $H^{1 / 2}([0, L])$ and the equivalence

$$
c_{1}\left\|\tilde{\psi}_{h}\right\|_{H^{1 / 2}(0, L)} \leq\left\|\psi_{h}\right\|_{H^{1 / 2}(0, L)} \leq c_{2}\left\|\tilde{\psi}_{h}\right\|_{H^{1 / 2}(0, L)}
$$

holds with $c_{1}=d, c_{2}=d^{-1}$ independent of $h$ and $\psi_{h}$.

(ii) The same is true if we replace $H^{1 / 2}(0, L)$ by $H_{00}^{1 / 2}(0, L)$ in (i).

Proof. We will make use of the following well-known norm equivalences (see 14. Chap. 1, Theorem 11.7]):

$$
\begin{gathered}
\int_{0}^{L} \int_{0}^{L}\left(\frac{\psi(x)-\psi(y)}{x-y}\right)^{2} d x d y+\int_{0}^{L} \psi(x)^{2} d x \\
\int_{0}^{L} \int_{0}^{L}\left(\frac{\psi(x)-\psi(y)}{x-y}\right)^{2} d x d y+\int_{0}^{L} \frac{1}{x(L-x)} \psi(x)^{2} d x \sim\|\psi\|_{H^{1 / 2}(0, L)}^{2},
\end{gathered}
$$

(i) Transformation of the integrals in (3.6) and application of (3.5) yield

$$
\begin{aligned}
& \left\|\psi_{h}\right\|_{H^{1 / 2}(0, L)}^{2} \sim \int_{0}^{L}\left(\psi_{h} \circ w_{h}\right)^{2} w_{h}^{\prime} d x \\
& \quad+\int_{0}^{L} \int_{0}^{L}\left(\frac{\psi_{h}\left(w_{h}(x)\right)-\psi_{h}\left(w_{h}(y)\right)}{x-y}\right)^{2}\left(\frac{x-y}{w_{h}(x)-w_{h}(y)}\right)^{2} w_{h}^{\prime}(x) w_{h}^{\prime}(y) d x d y \\
& \leq \frac{h_{\max }}{h_{\min }} \int_{0}^{L}\left(\psi_{h} \circ w_{h}\right)^{2} d x+\frac{h_{\max }^{2}}{h_{\min }^{2}} \int_{0}^{L} \int_{0}^{L}\left(\frac{\psi_{h} \circ w_{h}(x)-\psi_{h} \circ w_{h}(y)}{x-y}\right)^{2} d x d y \\
& \leq \frac{1}{d^{2}}\left\|\tilde{\psi}_{h}\right\|_{H^{1 / 2}(0, L)}^{2} .
\end{aligned}
$$

Similarly, we get $\left\|\psi_{h}\right\|_{H^{1 / 2}(0, L)} \geq d\left\|\tilde{\psi}_{h}\right\|_{H^{1 / 2}(0, L)}$.

(ii) We use representation (3.7) of the norm in $H_{00}^{1 / 2}(0, L)$ and follow the proof of (i). It remains to estimate the term

$$
\int_{0}^{L} \frac{\psi_{h}(x)^{2}}{x(L-x)} d x=\int_{0}^{L} \frac{\left(\psi_{h} \circ w_{h}(x)\right)^{2}}{x(L-x)} \frac{x(L-x)}{w_{h}(x)\left(L-w_{h}(x)\right)} w_{h}^{\prime}(x) d x .
$$

The fraction

$$
\frac{x(L-x)}{w_{h}(x)\left(L-w_{h}(x)\right)}=\frac{x}{w_{h}(x)-w_{h}(0)} \frac{L-x}{w_{h}(L)-w_{h}(x)}
$$

in (3.8) can be estimated with help of the Lipschitz property (3.5) which leads to the upper bound $h_{\text {mean }}^{2} / h_{\min }^{2}$ and the lower bound $h_{\text {mean }}^{2} / h_{\text {max }}^{2}$. For the weight in (3.8) we get the estimate

$$
d^{2} \leq \frac{h_{\min }^{2}}{h_{\max }^{2}} \leq \frac{x(L-x)}{w_{h}(x)\left(L-w_{h}(x)\right)} w_{h}^{\prime}(x) \leq \frac{h_{\max }^{2}}{h_{\min }^{2}} \leq d^{-2} .
$$


The lemma leads to an $L^{2}$-symmetric preconditioner which evaluates $\psi \circ w_{h}$ instead of $\psi$ :

Corollary 3.2. On $H_{00}^{1 / 2}(0, L)$, the operator $\mathcal{C}_{w_{h}} \psi=\frac{1}{w_{h}^{\prime} \circ w_{h}^{-1}}\left(-\Delta_{[0, L]}\right)^{1 / 2}\left(\psi \circ w_{h}\right) \circ$ $w_{h}^{-1}$ is spectrally equivalent to $\left(-\Delta_{[0, L]}\right)^{1 / 2}$ with equivalence constants uniform with respect to $h$.

Proof. For $\psi$ taken from the dense subspace $H_{0}^{1}(0, L)$ of $H_{00}^{1 / 2}(0, L)$ we derive by Lemma 3.1 and by an integral transformation

$$
\begin{aligned}
& \left(\left(-\Delta_{\Gamma_{i}}\right)^{1 / 2} \psi, \psi\right)_{L^{2}(0, L)} \sim\left(\left(-\Delta_{\Gamma_{i}}\right)^{1 / 2}\left(\psi \circ w_{h}\right), \psi \circ w_{h}\right)_{L^{2}(0, L)} \\
& =\int_{0}^{L}\left(-\Delta_{\Gamma_{i}}\right)^{1 / 2}\left(\psi \circ w_{h}\right)(x) \psi \circ w_{h}(x) d x \\
& =\int_{0}^{L} \frac{\left(\left(-\Delta_{\Gamma_{i}}\right)^{1 / 2}\left(\psi \circ w_{h}\right)\right) \circ w_{h}^{-1}(y)}{w_{h}^{\prime} \circ w_{h}^{-1}(y)} \psi(y) d y=\left(\mathcal{C}_{w_{h}} \psi, \psi\right)_{L^{2}(0, L)} .
\end{aligned}
$$

Note that in the discrete version

$$
\mathcal{C}_{w_{h}}^{h} \psi_{h}=\frac{1}{w_{h}^{\prime} \circ w_{h}^{-1}} \mathcal{C}^{h}\left(\psi_{h} \circ w_{h}\right) \circ w_{h}^{-1},
$$

$\mathcal{C}_{h}\left(\psi \circ w_{h}\right) \circ w_{h}^{-1}$ is simply the application of $\mathcal{C}_{h}$ to the discrete mesh values of $\psi_{h}$, and that $w_{h}^{\prime} \circ w_{h}^{-1}(x)$ evaluates to the local mesh size $h_{l o c}$ divided by $h_{\text {mean }}$.

The consequence of Corollary 3.2 is that for uniform boundary meshes we can use the preconditioners developed in Section 3.1 without loss of the independence of the condition number on the discretization parameter if we weight the result by $1 / h_{l o c}$. The generalization of the corollary to the Neumann case is obvious. For $\sigma>0$, the norm equivalence (3.4) leads directly to the preconditioner

$$
\sqrt{\sigma} I+\mathcal{C}_{w_{h}}^{h}
$$

3.3. Preconditioners on Gauss-Lobatto (GL) boundary meshes. If spectral Chebyshev solvers [2, 3] are used on some of the subdomains, they require a Chebyshev-Gauss-Lobatto mesh. Its restriction to an interface $\Gamma_{i}$ is a onedimensional Chebyshev-Gauss-Lobatto mesh. If we identify $\Gamma_{i}$ with the interval $[-1,1]$, the Gauss-Lobatto mesh points are given by

$$
x_{k}=\cos \frac{\pi k}{N}, \quad k=0, \ldots, N .
$$

Clearly, this mesh is not uniform, as $h_{\min }=x_{N-1}-x_{N}=O\left(h_{\max }^{2}\right)$, i.e., $h_{\max } / h_{\min }$ $\rightarrow \infty$ for $N \rightarrow \infty$. So Lemma 3.1 is useless for this kind of mesh. Numerical tests using interpolation of the discrete values given on the GL mesh to an equidistant mesh gave unsatisfactory results 6 Instead of this, an approach similar to Section 3.2 is investigated.

Observing that the Gauss-Lobatto grid values of $\psi$ on $[-1,1]$ are identical with the equidistant grid values of $\psi \circ \cos$ on $[0, \pi]$, it is obvious that the numerical evaluation of $\left(-\Delta_{\Gamma_{i}}\right)^{1 / 2}(\psi \circ \cos )$ can be done following Section 3.1. So we have to

\footnotetext{
${ }^{6}$ It is well known that local interpolation of data can strongly perturbate Fourier analysis.
} 
express $\left\|\left(-\Delta_{\Gamma_{i}}\right)^{1 / 2} \varphi\right\|_{L^{2}}$ in terms of $\left\|\left(-\Delta_{\Gamma_{i}}\right)^{1 / 2}(\varphi \circ \cos )\right\|_{L^{2}}$. To get this connection, we make use of the interpolation theory of weighted Sobolev-Slobodeckij spaces.

Let us consider the case of Dirichlet boundary conditions at $y= \pm 1$, i.e., $\psi \in$ $H_{00}^{1 / 2}(-1,1)$.

Lemma 3.3. For $\psi \in H_{00}^{1 / 2}(-1,1), \psi \circ \cos \in H_{00}^{1 / 2}(0, \pi)$ and the equivalence of the norms

$$
\|\psi\|_{H_{00}^{1 / 2}(-1,1)} \sim\|\psi \circ \cos \|_{H_{00}^{1 / 2}(0, \pi)}
$$

holds.

Proof. It is sufficient to show the equivalence (3.9) on a dense subspace of $H_{00}^{1 / 2}(-1,1)$. So let $\psi$ be taken from $H_{0}^{1}(-1,1)$. Let us define the weighted norms

$$
\|\psi\|_{L_{w}^{2}(a, b)}^{2}=\int_{a}^{b} w|\psi|^{2} d y, \quad\|\psi\|_{H_{w_{1}, w_{2}}^{1}(a, b)}^{2}=\int_{a}^{b} w_{1}|\nabla \psi|^{2} d y+\int_{a}^{b} w_{2}|\psi|^{2} d y
$$

and let $L_{w}^{2}(a, b)$ and $H_{0, w_{1}, w_{2}}^{1}(a, b)$ be the closures of $C_{0}^{\infty}(a, b)$ with respect to these norms. By integral transformation it is easy to see that for $w(y):=(\sin y)^{-1}$

$$
\|\psi\|_{L^{2}(-1,1)}=\|\psi \circ \cos \|_{L_{w-1}^{2}(0, \pi)}, \quad\|\psi\|_{H_{0}^{1}(-1,1)}=\|\psi \circ \cos \|_{H_{w, w}^{1}-1}(0, \pi) .
$$

Therefore, the equivalence

$$
\left.\|\psi\|_{\left[L^{2}(-1,1), H_{0}^{1}(-1,1)\right]_{1 / 2}} \sim\|\psi \circ \cos \|_{\left[L_{w^{-1}}^{2}(0, \pi), H_{w, w}^{1}-1\right.}(0, \pi)\right]_{1 / 2}
$$

holds. By well-known interpolation results ([19, p. 277]), the weight functions $w$ and $w^{-1}$ on the right-hand side extinguish each other:

$$
\|\psi \circ \cos \|_{\left[L_{w-1}^{2}(0, \pi), H_{w, w}^{1}(0, \pi)\right]_{1 / 2}} \sim\|\psi \circ \cos \|_{H_{00}^{1 / 2}(0, \pi)} .
$$

Corollary 3.4. On $H_{00}^{1 / 2}(-1,1)$, the operator $\mathcal{C}_{G L}$,

$$
\mathcal{C}_{G L} \psi(s)=\frac{1}{\left(1-s^{2}\right)^{1 / 2}}\left(\left(-\Delta_{\Gamma_{i}}\right)^{1 / 2}(\psi \circ \cos ) \circ \arccos \right)(s),
$$

is spectrally equivalent to $\left(-\Delta_{\Gamma_{i}}\right)^{1 / 2}$.

Proof. Analogous to the proof of Corollary 3.2 we get

$$
\begin{aligned}
\left(\left(-\Delta_{\Gamma_{i}}\right)^{1 / 2} \psi, \psi\right)_{L^{2}(-1,1)} & \sim\left(\left(-\Delta_{\Gamma_{i}}\right)^{1 / 2}(\psi \circ \cos ), \psi \circ \cos \right)_{L^{2}(0, \pi)} \\
& =\left(\mathcal{C}_{G L} \psi, \psi\right)_{L^{2}(-1,1)} .
\end{aligned}
$$

As a discretization, we consequently use

$$
\mathcal{C}_{G L}^{h} \psi(s)=\frac{1}{\left(1-s^{2}\right)^{1 / 2}}\left(\mathcal{C}^{h}(\psi \circ \cos ) \circ \arccos \right)(s)
$$

where $\mathcal{C}^{h}$ is a discretization of $\left(-\Delta_{[-1,1]}\right)^{1 / 2}$ in accordance with Section [3.1. So up to a weight function, we can use the equidistant mesh preconditioner $\mathcal{C}^{h}$ also on the Gauss-Lobatto mesh, at least for Dirichlet conditions on $\partial \Omega \cap \Gamma_{i}$. A similar proof for the case of Neumann conditions at $\Omega \cap \Gamma_{i}$ seems difficult due to the lack of 
interpolation results for $H^{1 / 2}$ comparable to (3.11) for $H_{00}^{1 / 2}$. However, numerical results using

$$
\mathcal{C}_{G L, N m}^{h} \psi(s):=\left(1-s^{2}\right)^{-1 / 2}\left(\mathcal{C}_{N m}^{h}(\psi \circ \cos ) \circ \arccos \right)(s)
$$

show that this technique is also efficient in the Neumann case.

An application of this method to other (nonuniform boundary meshes) is possible for Dirichlet conditions. For the generalization to the case $\sigma>0$, see the remark at the end of Section 3.2

3.4. Possible generalizations to 3-dimensional problems. If $\Omega \subset \mathbb{R}^{3}$ and if the interfaces $\Gamma_{i}$ are squares (as is usually the case when spectral methods are used; the $\Omega_{i}$ are cuboids), the application of the ideas of the previous sections for the discretization of the preconditioner is straightforward. Even if the geometry is deviant and spectral methods are used in combination with mappings onto squares/cubes, the methods can be applied. The same is true for finite difference solvers if the boundary data are given or can be mapped on a Cartesian mesh.

If the boundary values are given on an unstructured FE boundary mesh, it should be possible to generalize the results of Section 3.1 .2 by hierarchical bases/multigrid methods [4].

\section{A COMparison of the CONDition number of CGBI AND FETI FOR A MODEL PROBLEM}

In Section 2.3 it was proven that the condition number $\kappa$ of the operator $\mathcal{C} \mathcal{A}$ (and consequently, the convergence rate of CGBI) is only dependent on the norm of the local trace and prolongation operators but is independent of the number of subdomains and the size of the global domain. However, it seems worthwhile to retrieve more explicit expressions for $\kappa$. These can be found if we restrict ourselves to a simplified geometry. The same investigation can be done for FETI with the so-called FETI Dirichlet preconditioner proposed in 9 10. Note that other preconditioners, e.g., the one proposed by Klawonn and Widlund 12 coincide with the classical Dirichlet preconditioner for the problems considered in this paper. We should mention that a "cheap" interface-based preconditioner called the "lumped preconditioner" was already proposed for FETI, but its performance was rather unsatisfactory ([8, Tab. 1] and [10, p. 1967]).

As in Section 2, we use the setting of functional spaces. Note that the possibility of using the nondiscretized operators also for the analysis of FETI is a consequence of our restriction to domain decompositions without interior cross-points. In a more general setting, the FETI condition number depends slightly on the discretization parameter [17, 18].

\subsection{Condition number for CGBI on a simply-shaped domain.}

Theorem 4.1. Let $\Omega=(0, S) \times(0, L)$, be a rectangle, let $p$ be an even number and let each subdomain $\Omega_{i}, i=1, \ldots, p$, be a rectangle of size $S / p \times L$ (cf. Figure 2 , top). Let us assume (i) Dirichlet or (ii) Neumann conditions on $y=0$ and $y=L$ and periodic boundary conditions at $x=0$ and $x=S$. Let us denote the subdomain aspect ratio $r=S /(p L)$. Then, 


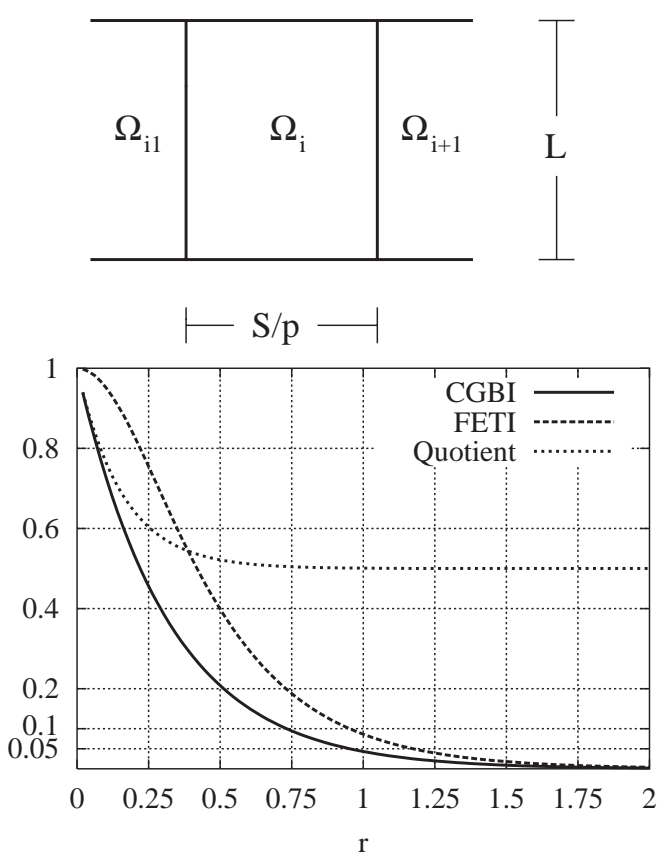

Figure 2. Top: Definition of the subdomain aspect ratio $r=$ $(S / p) / L$. Bottom: Comparison of the convergence rate $q$ (error reduction per iteration step) for CGBI and FETI and the quotient of both rates as a function of the subdomain aspect ratio $r$.

(a) the operator $\mathcal{A}(2.10)$ has the eigenvalues

$$
\mu_{k, m}=\frac{2 L}{\sqrt{\sigma L^{2}+\pi^{2} k^{2}}} \frac{\cosh r \sqrt{\sigma L^{2}+\pi^{2} k^{2}}-\cos \frac{2 \pi m}{p}}{\sinh r \sqrt{\sigma L^{2}+2 \pi^{2} k^{2}}}, \quad m=0, \ldots, \frac{p}{2}, k \in \mathbb{N} ;
$$

(b) the preconditioned operator $\mathcal{C}^{1 / 2} \mathcal{A C}^{1 / 2}$ with $\mathcal{C}=\left(\sigma I-\Delta_{\Gamma}\right)^{1 / 2}$ in case (i) and $\mathcal{C}=\left(\sigma I-\Delta_{\Gamma_{N m}}\right)^{1 / 2}$ in case (ii) has the eigenvalues

$$
\lambda_{k, m}=2 \frac{\cosh r \sqrt{\sigma L^{2}+\pi^{2} k^{2}}-\cos \frac{2 \pi m}{p}}{\sinh r \sqrt{\sigma L^{2}+\pi^{2} k^{2}}}, \quad m=0, \ldots, \frac{p}{2}, k \in \mathbb{N} ;
$$

(c) for the condition number the equality

$$
\kappa\left(\mathcal{C}^{1 / 2} \mathcal{A C}^{1 / 2}\right)=\frac{\cosh \left(r \sqrt{\sigma L^{2}+\pi^{2}}\right)+1}{\cosh \left(r \sqrt{\sigma L^{2}+\pi^{2}}\right)-1}=\frac{2}{\cosh \left(r \sqrt{\sigma L^{2}+\pi^{2}}\right)-1}+1
$$

holds which can be estimated by

$$
\kappa \leq \frac{2}{\cosh (r \pi)-1}+1
$$

d) the condition number has the following asymptotic behavior:

For fixed $\sigma \in[0, \infty), \lim _{r \rightarrow \infty} \kappa=1$, and for $r \longrightarrow 0, \kappa=O\left(r^{-2}\right)$; for fixed $r \in(0, \infty), \kappa$ is decaying monotonically for $\sigma \rightarrow \infty$, and $\lim _{\sigma \rightarrow \infty} \kappa=1$. 
Proof. We consider the boundary at $x=S, x=0$ as an interface $\Gamma_{p}$ between $\Omega_{p}$ and $\Omega_{1}$. For short let us set

$$
\begin{gathered}
K=r \sqrt{\sigma L^{2}+\pi^{2} k^{2}}, \\
d_{i}=\sin \frac{2 \pi i m}{p}, \quad s_{i}=\frac{\sin \frac{2 \pi(i+1) m}{p}-\sin \frac{2 \pi i m}{p} \cosh K}{\sinh K}, \\
\bar{d}_{i}=\cos \frac{2 \pi i m}{p}, \quad \bar{s}_{i}=\frac{\cos \frac{2 \pi(i+1) m}{p}-\cos \frac{2 \pi i m}{p} \cosh K}{\sinh K}, \quad i=1, \ldots, p .
\end{gathered}
$$

(a) Let us define $\varphi_{k, m}, \bar{\varphi}_{k, m}: \Gamma \rightarrow \mathbb{R}$ by

$$
\left.\varphi_{k, m}\right|_{\Gamma_{i}}=d_{i} \sin \frac{\pi k y}{L},\left.\quad \bar{\varphi}_{k, m}\right|_{\Gamma_{i}}=\bar{d}_{i} \sin \frac{\pi k y}{L},
$$

$y \in(0, L)$, for boundary condition (i) and

$$
\left.\varphi_{k, m}\right|_{\Gamma_{i}}=d_{i} \cos \frac{\pi k y}{L},\left.\quad \bar{\varphi}_{k, m}\right|_{\Gamma_{i}}=\bar{d}_{i} \cos \frac{\pi k y}{L}
$$

for boundary condition (ii). We will prove that $\varphi_{k, m}$ for $m=1, \ldots, p / 2-1$ and $\bar{\varphi}_{k, m}$ for $m=0, \ldots, p / 2$ are eigenfunctions of $\mathcal{A}$, i.e.,

$$
\left[v\left(\varphi_{k, m}\right)\right]=\mu_{k, m} \varphi_{k, m}, \quad\left[v\left(\bar{\varphi}_{k, m}\right)\right]=\mu_{k, m} \bar{\varphi}_{k, m} .
$$

Let us focus on case (i).

Obviously, the functions $v_{k, m}=\left(v_{k, m}^{1}, \ldots, v_{k, m}^{p}\right)$ and $\bar{v}_{k, m}=\left(\bar{v}_{k, m}^{1}, \ldots, \bar{v}_{k, m}^{p}\right)$, where

$$
\begin{aligned}
& v_{k, m}^{i}(x, y)=\left(\frac{K}{r L}\right)^{-1}\left(d_{i-1} \sinh \frac{x K}{r L}+s_{i-1} \cosh \frac{x K}{r L}\right) \sin \frac{\pi k y}{L}, \\
& \bar{v}_{k, m}^{i}(x, y)=\left(\frac{K}{r L}\right)^{-1}\left(\bar{d}_{i-1} \sinh \frac{x K}{r L}+\bar{s}_{i-1} \cosh \frac{x K}{r L}\right) \sin \frac{\pi k y}{L},
\end{aligned}
$$

are solutions of $(\sigma I-\Delta) v^{i}=0$ on $\Omega_{i}$ for arbitrary $d_{i-1}, s_{i-1} \in \mathbb{R}$. Let us identify $\Omega_{i}=(0, r L) \times(0, L)$. It is easy to check that for $d_{i}, s_{i}, \bar{d}_{i}, \bar{s}_{i}$ from (4.4), each $v_{k, m}^{i}$, $\bar{v}_{k, m}^{i}$ also meets the boundary conditions of problem (2.6) with interface boundary condition $\varphi=\varphi_{k, m}, \varphi=\bar{\varphi}_{k, m}$, respectively. A computation of the jumps $\left[v\left(\varphi_{k, m}\right)\right]$, $\left[v\left(\bar{\varphi}_{k, m}\right)\right]$ shows that (4.5) holds.

(b) The functions $\varphi_{k, m}, \bar{\varphi}_{k, m}$ defined in the proof of (a) are obviously also eigenfunctions of $\mathcal{C}$, and the corresponding eigenvalues are $\tilde{\mu}_{k, m}=\sqrt{\sigma+\frac{\pi^{2} k^{2}}{L^{2}}}$. Hence, the $\varphi_{k, m}$ are also eigenfunctions of $\mathcal{C}^{1 / 2} \mathcal{A C}^{1 / 2}$ and (4.1) follows.

(c) (4.1) takes its maximum (minimum) value for $m=p / 2, k=1(m=0, k=1)$. $\kappa\left(\mathcal{C}^{1 / 2} \mathcal{A C}^{1 / 2}\right)=\lambda_{1, p / 2} / \lambda_{1,0}$ yields (4.2).

(4.3) follows from the monotonicity of the right-hand side of (4.2) considered as a function of $\sigma$.

(d) A series expansion of the cosh in the right-hand side of (4.2) shows $\kappa=$ $O\left(r^{-2}\right)$ for $r \rightarrow 0$. The behavior for $r \rightarrow \infty$ and for $\sigma \rightarrow \infty$ is obvious.

4.2. Condition number for FETI on a simply-shaped domain. Let us consider now the condition number of FETI for the same simply-shaped domain with the same substructuring as in Theorem 4.1 with boundary conditions (i). As there 
are no interior cross-points, FETI simplifies to solve the same problem $[v(\varphi)]=$ $-[w] \sqrt{7}$ i.e., in the setting of functional spaces, the operator of FETI coincides with the CGBI operator $\mathcal{A}$. The difference lies in the preconditioning. The most usual FETI preconditioner for our problem is based on the solution of the Dirichlet problem

$$
\begin{aligned}
\mathcal{L} \tilde{v}_{i} & =0 \text { in } \Omega_{i} \\
\tilde{v}_{i} & =\psi_{i-1} \text { on } \Gamma_{i-1}, \\
\tilde{v}_{i} & =\psi_{i} \text { on } \Gamma_{i} \\
\tilde{v}_{i} & =0 \text { on } \partial \Omega_{i} \cap \partial \Omega .
\end{aligned}
$$

The preconditioner (which is called the Dirichlet preconditioner [9]) is the mapping of a $\psi \in H_{00}^{1 / 2}(\Gamma)$ to the jump of the Neumann trace $[\tilde{v}(\psi)]^{N m}$ defined by $\left.[\tilde{v}(\psi)]^{N m}\right|_{\Gamma_{i}}=\partial \tilde{v}_{i}(\psi) / \partial \nu_{i}-\partial \tilde{v}_{i+1}(\psi) / \partial \nu_{i}$. Let us denote this preconditioner by $\mathcal{C}_{F E T I}$.

Theorem 4.2. Under the assumptions of Theorem 4.1 the condition number $\kappa\left(\mathcal{C}_{F E T I}^{1 / 2} \mathcal{A C}_{F E T I}\right)$ of the nondiscretized FETI method with the Dirichlet preconditioner is equal to the square of the $C G B I$ condition number $\kappa\left(\mathcal{C}^{1 / 2} \mathcal{A C}^{1 / 2}\right)$.

Proof. Let $d_{i}, s_{i}, \bar{d}_{i}, \bar{s}_{i}$ be defined as in the proof of Theorem 4.1. Similar to the proof of Theorem 4.1 (a) one sees that $\psi_{k, m}$ for $m=1, \ldots, p / 2-1$ and $\bar{\psi}_{k, m}$ for $m=$ $0, \ldots, p / 2$ with $\left.\psi_{k, m}\right|_{\Gamma_{i}}=d_{i} \sin \frac{\pi k y}{L},\left.\bar{\psi}_{k, m}\right|_{\Gamma_{i}}=\bar{d}_{i} \sin \frac{\pi k y}{L}$ are eigenfunctions of the operator $\mathcal{C}_{F E T I}$ for case (i): To check this, one uses the fact that the corresponding solutions $\tilde{v}\left(\psi_{k, m}\right), \tilde{v}\left(\bar{\psi}_{k, m}\right)$ of problem (4.6) are

$$
\begin{aligned}
& \tilde{v}_{i}\left(\psi_{k, m}\right)=\left(s_{i-1} \sinh \frac{x K}{r L}+d_{i-1} \cosh \frac{x K}{r L}\right) \sin \frac{k \pi y}{L}, \\
& \tilde{v}_{i}\left(\bar{\psi}_{k, m}\right)=\left(\bar{s}_{i-1} \sinh \frac{x K}{r L}+\bar{d}_{i-1} \cosh \frac{x K}{r L}\right) \sin \frac{k \pi y}{L}
\end{aligned}
$$

on $\Omega_{i}, \Omega_{i}$ identified with $(0, r L) \times(0, L)$. The corresponding Neumann jumps $\left[\tilde{v}\left(\psi_{k, m}\right)\right]^{N m},\left[\tilde{v}\left(\bar{\psi}_{k, m}\right)\right]^{N m}$ are equal to $\mu_{k, m}^{F E T I} \psi_{k, m}, \mu_{k, m}^{F E T I} \bar{\psi}_{k, m}$, respectively. So the eigenvalues of $\mathcal{C}_{F E T I}$ are

$$
\mu_{k, m}^{F E T I}=\frac{2 \sqrt{\sigma L^{2}+\pi^{2} k^{2}}}{L} \frac{\cosh r \sqrt{\sigma L^{2}+\pi^{2} k^{2}}-\cos \frac{2 \pi m}{p}}{\sinh r \sqrt{\sigma B^{2}+\pi^{2} k^{2}}}, \quad m=0, \ldots, \frac{p}{2}, k \in \mathbb{N} .
$$

Since the eigenspaces of $\mathcal{A}$ and $\mathcal{C}_{F E T I}$ coincide, the eigenvalues of the operator $\mathcal{C}_{F E T I}^{1 / 2} \mathcal{A} \mathcal{C}_{F E T I}^{1 / 2}$ are

$$
\lambda_{k, m}^{F E T I}=\mu_{k, m} \mu_{k, m}^{F E T I}=4\left(\frac{\cosh r \sqrt{\sigma L^{2}+\pi^{2} k^{2}}-\cos \frac{\pi m}{p}}{\sinh r \sqrt{\sigma B^{2}+2 \pi^{2} k^{2}}}\right)^{2},
$$

and the condition number is

$$
\kappa\left(\mathcal{C}_{F E T I}^{1 / 2} \mathcal{A C} \mathcal{C}_{F E T I}^{1 / 2}\right)=\left(\frac{\cosh r \sqrt{\sigma L^{2}+\pi^{2}}+1}{\cosh r \sqrt{\sigma L^{2}+\pi^{2}}-1}\right)^{2}=\kappa\left(\mathcal{C}^{1 / 2} \mathcal{A C}^{1 / 2}\right)^{2} .
$$

\footnotetext{
${ }^{7}$ In the FETI community, the Neumann interface condition $\varphi$ is usually considered as a Lagrangian multiplier for a constrained minimization problem [8].
} 
Let us consider the CGBI condition number $\kappa$ in (4.2) and the FETI condition number (4.7) as a function of $r(\sigma=0)$. If we assume the asymptotic CG error reduction rate $q$ to be $q=(\sqrt{\kappa}-1) /(\sqrt{\kappa}+1)$, Figure 2 shows the resulting error reduction rate $q$ as a function of $r$. So the CGBI error reduction rate for the model problem appears to be significantly smaller than that of the preconditioned FETI method. More precisely, the quotient of the convergence rate is about $1 / 2$ for $r$ not too small. The deterioration of the convergence rates for $r \rightarrow 0$ is worse for FETI than for CGBI.

Remark 4.3 (The choice of boundary conditions). In Theorem 4.1, similar results for Neumann and for Dirichlet instead of periodic conditions at $x=0, x=S$ and arbitrary $p \geq 2$ can be proved ([13, p. $74 \mathrm{ff}$.$] ). In Theorem 4.2$ the eigenspaces of $\mathcal{A}$ and of $C_{F E T I}$ differ under those boundary conditions, making an analysis more difficult.

\section{Numerical Results}

In the following we will verify the theoretical results of the previous chapters by numerical results. We will use the simple geometry of Theorem 4.1 with quadratic subdomains $(r=1)$, Dirichlet conditions on $\partial \Omega$ and $\sigma=0$. Some numerical results for different $\sigma, r$ and Neumann conditions can be found in [13.

Figure 3 shows the convergence of CGBI for the Poisson equation on a domain $\Omega=(0,8) \times(0,1)$ divided into 8 quadratic subdomains. An FE solver (linear elements) on a regular mesh with $512 \times 512$ nodes per subdomain was used. The right-hand side of the problem was chosen such that the solution is in $C^{\infty}(\bar{\Omega})$. In order to reveal the worst-case behavior of CGBI, the CG iteration was started with a (piecewise linear) $\varphi$ with nodal values which were chosen at random between \pm 1 . The diagrams show the $H^{1}$-error (with respect to each subdomain), the $L^{2}(\Omega)$-, $L^{\infty}(\Omega)$-error for $v(\varphi)$, and the $H^{-1 / 2}(\Gamma)$-error for $\varphi$. As the theory of CG predicts, we see a monotonous decay for the $H^{1}$-norm. Furthermore, we observe that the $H^{-1 / 2}(\Gamma)$-error for $\varphi$ is very close, which indicates that the constants $c_{1}, c_{2}$ in (2.13)-2.16) are not far from 1, a fact that was already predicted by Theorem 4.1 The bottom part of Figure 3 demonstrates the efficiency of the spectral preconditioner proposed in Section 3.1.1: The preconditioned CGBI only needs 3 iteration steps to reduce the error by $10^{-4}$, and 4 iteration steps instead of about 60 for the unpreconditioned to reach the finite element accuracy of a sequential solver. In order to make a comparison to earlier computational results for FETI, we also compute the residual: CGBI requires only 5 iteration steps to reduce the $L^{2}(\Omega)$ residual by at least $10^{-6}$ (see Table11). Note the close coincidence of the mean error reduction rate of Table 1 with the theoretical result of Figure 2 at $r=1$. FETI with the Dirichlet preconditioner on the same geometry requires 9-11 iteration steps for the same reduction of the residual ([9, Chapter 4.2]). The fact that CGBI needs fewer iteration steps for this problem was also predicted in Section 4.2 (Figure 2 . bottom). The cpu time for the CGBI preconditioning covers about $1 \%$ of the computational time of the unpreconditioned CGBI, while the Dirichlet preconditioner should require (for large $N$ ) almost $100 \%$ (about $80 \%$ for an example given in [10] p. 1967] of the computational time of the unpreconditioned FETI).

In Figure 4 the same problem with the same domain decomposition is computed for different values for the discretization parameter $N(N \times N$ being the degrees of freedom on each subdomain). For unpreconditioned CGBI, the number of iteration 


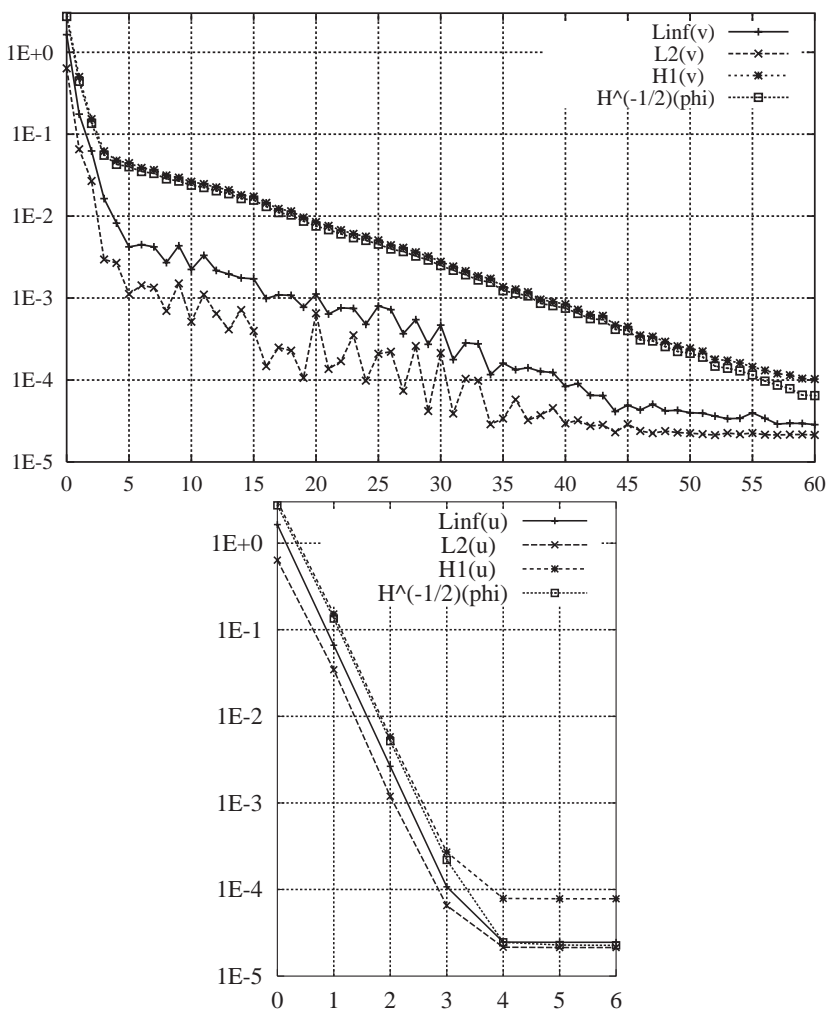

FiguRE 3. Convergence of CGBI in different norms. Horizontal axis: Number of iteration step. Domain $\Omega=(0,8) \times(0,1)$ divided into 8 quadratic subdomains. FE solver on subdomains, regular mesh with $512 \times 512$ nodes per subdomain. Top: Without preconditioning. Bottom: With spectral preconditioner (Section 3.1.1)

steps depends strongly on $N$. For the spectral preconditioner, three iteration steps are sufficient.

TABLE 1. Relative residual of equation (1.1) with respect to the $L^{2}(\Omega)$-norm after 5 CGBI steps (preconditioned) and mean reduction of the residual per CGBI step. After 5 steps, the residual is reduced by more than $10^{-6}$. Quadratic subdomains $(r=1)$.

\begin{tabular}{|r|l|l|l|}
\hline$p$ & $N$ & $\begin{array}{l}\text { rel. residual } \\
\text { after 5 steps }\end{array}$ & $\begin{array}{l}\text { mean red. } \\
\text { per step }\end{array}$ \\
\hline \hline 2 & 64 & $8.33 \mathrm{E}-12$ & 0.006 \\
4 & 64 & $7.68 \mathrm{E}-11$ & 0.009 \\
6 & 64 & $3.11 \mathrm{E}-9$ & 0.020 \\
8 & 64 & $1.70 \mathrm{E}-7$ & 0.044 \\
32 & 64 & $1.53 \mathrm{E}-7$ & 0.043 \\
128 & 64 & $1.52 \mathrm{E}-7$ & 0.043 \\
\hline
\end{tabular}

\begin{tabular}{|l|r|l|l|}
\hline$p$ & $N$ & $\begin{array}{l}\text { rel. residual } \\
\text { after 5 steps }\end{array}$ & $\begin{array}{l}\text { mean red. } \\
\text { per step }\end{array}$ \\
\hline \hline 8 & 16 & $1.79 \mathrm{E}-7$ & 0.044 \\
8 & 64 & $1.70 \mathrm{E}-7$ & 0.044 \\
8 & 256 & $1.69 \mathrm{E}-7$ & 0.044 \\
\hline
\end{tabular}




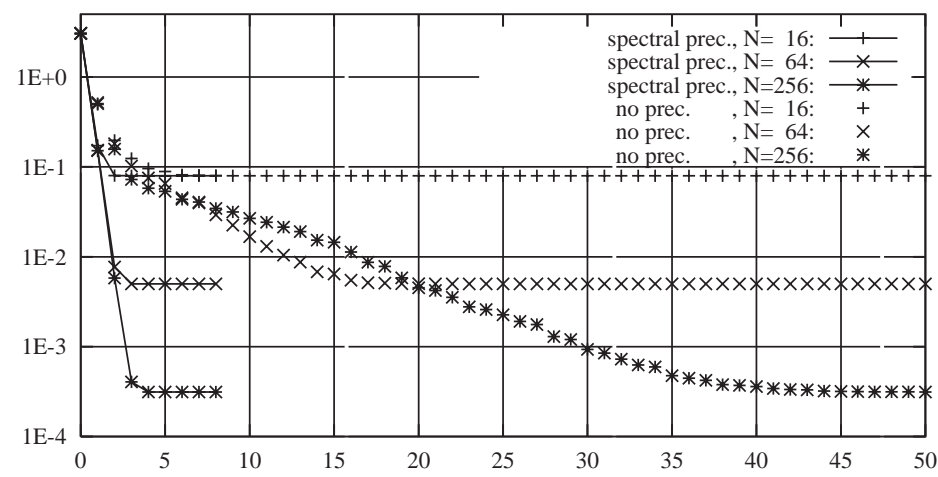

FiguRE 4. Convergence of CGBI with respect to $H^{1}(\Omega)$-norm for different discretization parameters $N$. All data as in Figure 3

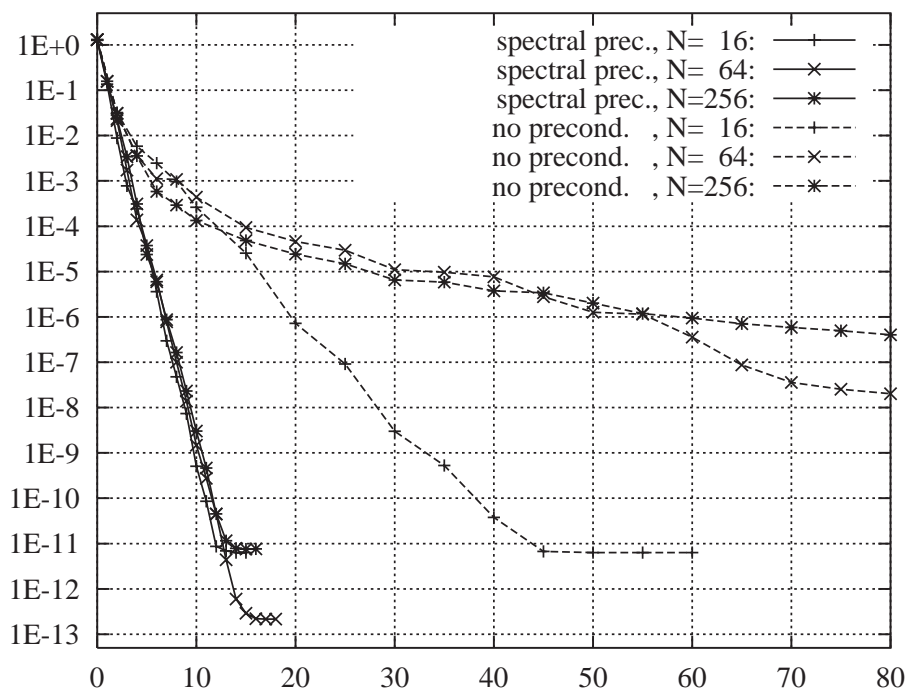

Figure 5. The spectral preconditioner on Chebyshev-GaussLobatto boundary mesh. Poisson equation on six spectral subdomains. $L^{\infty}(\Omega)$-error in dependence of the CGBI step. Different discretization parameters $N$.

Figure 5 shows the convergence of CGBI for 4 subdomains with the spectral collocation Chebyshev solver on each subdomain. Since the boundary mesh on the interfaces is a Chebyshev-Gauss-Lobatto mesh, we use the preconditioner $\mathcal{C}_{G L}^{h}$ (3.13). Since the derivation of this preconditioner requires the use of the equivalence (3.11), the condition number increases by the ratio of the equivalence constants in (3.11). Therefore we can observe that the convergence speed for $\mathcal{C}_{G L}^{h}$ is slightly lower than that of $\mathcal{C}^{h}$ on an equidistant boundary mesh (Figure 4). However, it is still about one power of 10 per CG step, and it is still independent of $N$.

Figure 6] shows a comparison of different discretizations of the preconditioner $\mathcal{C}_{G L}$ for a Chebyshev-Gauss-Lobatto mesh. The figure shows the performance of the transformation (3.13) together with the discretization $\mathcal{C}^{h}$ (spectral), $\mathcal{C}_{M}$ (multidiagonal), $\mathcal{C}_{t r i}$ (tridiagonal), respectively (Section 3.1). Among these, the 


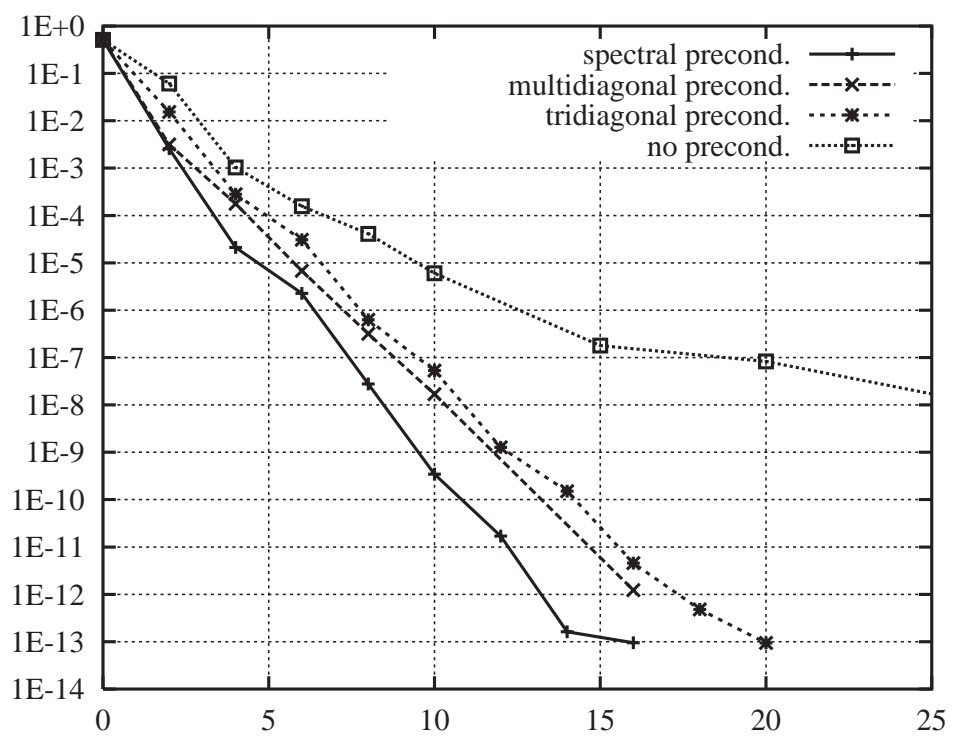

FiguRE 6. $L^{2}(\Omega)$-CGBI convergence rate for different preconditioners on Chebyshev-Gauss-Lobatto boundary mesh. Six spectral subdomains, $N=64$.

spectral version performs best with an error reduction of one power of 10 per CG step. Another preconditioner especially optimized for Gauss-Lobatto boundary meshes will be proposed in a forthcoming paper.

\section{Conclusion And outlook}

CGBI turned out to be an efficient algorithm for domain decomposition without interior cross-points. The CGBI interface preconditioners cause negligible computational costs. Nevertheless, even smaller condition numbers than for the Dirichletpreconditioned FETI method could be observed, making CGBI an efficient tool for computations on domains with large aspect ratio.

The efficient application of interface preconditioners for the more general case of domain decomposition with interior cross-points is presently an open question. However, first computational results for this situation are promising: For CGBI on domain decompositions with interior cross-points we found a reduction of the residual of about $10^{-6}$ within 18 iteration steps 8 Hence, the convergence rate is at least not worse than that of the FETI method in [9, Chapter 4.2], while the computational costs of the boundary preconditioner are much lower than that of a Dirichlet preconditioner.

Other possible extensions of the applicability of CGBI (even without inner crosspoints) could be the construction of discretizations of the interface preconditioners for complex 3-dimensional problems, like channel systems with bifurcations, where the domains and the interfaces are discretized by unstructured finite element meshes (Section 3.4).

\footnotetext{
${ }^{8} \mathrm{~A}$ checkerboard domain decomposition into $p \times p$ subdomains, $p=4,8,16,32$, and $16 \times 16$ nodes per subdomain were used.
} 


\section{APPENDIX}

Since the author could not find a proof of Lemma 2.1 (which is a slight generalization of the well-known trace theorem) in the literature, a proof is given here:

Proof. For $\sigma=0, T_{i}$ and $P_{\sigma, i}$ are continuous operators, which is a consequence of the well-known trace theorem and the Poincaré inequalities in $\left\{u \in H^{1}\left(\Omega_{i}\right)|u|_{\partial \Omega_{i} \backslash \Gamma_{i}}=\right.$ $0\}, H_{00}^{1 / 2}\left(\Gamma_{i}\right)$. Therefore it is sufficient to prove the statement of Lemma 2.1 for $\sigma \in(0, \infty)$.

In Part 1 of the following proof we consider the situation of a half space. In Part 2 we generalize the results to $\Omega_{i}$ being a cuboid. A further generalization to bounded domains with Lipschitz boundary can be obtained by the technique of local maps.

Part 1: First let us consider the situation of a half space $\Omega=\left\{x_{1}>0\right\} \times \mathbb{R}^{n-1}$, $\Gamma=\partial \Omega=\left\{x_{1}=0\right\} \times \mathbb{R}^{n-1}$.

(a) For $\sigma \in(0, \infty)$ and both for functions $u \in H^{1}(\Omega)$ and $u \in H^{1 / 2}(\Gamma)$ we define $\tilde{u}(x)=u\left(\sigma^{-1 / 2} x\right)$. The (semi-)norms of $u$ scale as follows:

$$
\begin{array}{ll}
\|\tilde{u}\|_{L^{2}(\Omega)}^{2}=\sigma^{\frac{n}{2}}\|u\|_{L^{2}(\Omega)}^{2}, & \|\nabla \tilde{u}\|_{L^{2}(\Omega)}^{2}=\sigma^{\frac{n-2}{2}}\|\nabla u\|_{L^{2}(\Omega)}^{2}, \\
\|\tilde{u}\|_{L^{2}(\Gamma)}^{2}=\sigma^{\frac{n-1}{2}}\|u\|_{L^{2}(\Gamma)}^{2}, & \left\|\left(-\Delta_{\Gamma}\right)^{1 / 4} \tilde{u}\right\|_{L^{2}(\Gamma)}^{2}=\sigma^{\frac{n-2}{2}}\left\|\left(-\Delta_{\Gamma}\right)^{1 / 4} u\right\|_{L^{2}(\Gamma)}^{2} .
\end{array}
$$

Let $T: H^{1}(\Omega) \rightarrow H^{1 / 2}(\Gamma)$ be the well-known trace operator. Obviously $(T u)^{r}=T \tilde{u}$ holds. Using the representation (2.3) of the norm $\|\cdot\|_{\sigma, \frac{1}{2}, \Gamma}$ and the scaling of the (semi-)norms of $T u$, we obtain

$$
\begin{aligned}
\|T u\|_{\sigma, \frac{1}{2}, \Gamma}^{2} & \sim \sigma^{\frac{1}{2}}\|T u\|_{L^{2}(\Gamma)}^{2}+\left\|\left(-\Delta_{\Gamma}\right)^{1 / 4} T u\right\|_{L^{2}(\Gamma)}^{2} \\
& =\sigma^{1-\frac{n}{2}}\left(\left\|(T u)^{2}\right\|_{L^{2}(\Gamma)}^{2}+\left\|\left(-\Delta_{\Gamma}\right)^{1 / 4}(T u)^{r}\right\|_{L^{2}(\Gamma)}^{2}\right) \\
& =\sigma^{1-\frac{n}{2}}\left(\|T \tilde{u}\|_{L^{2}(\Gamma)}^{2}+\left\|\left(-\Delta_{\Gamma}\right)^{1 / 4} T \tilde{u}\right\|_{L^{2}(\Gamma)}^{2}\right) .
\end{aligned}
$$

We proceed by using the well-known Trace Theorem $(\sigma=1)$ and by using the norm scaling again:

$$
\|T u\|_{\sigma, \frac{1}{2}, \Gamma}^{2} \leq c \sigma^{1-\frac{n}{2}}\left(\|\tilde{u}\|_{L^{2}(\Omega)}^{2}+\|\nabla \tilde{u}\|_{L^{2}(\Omega)}^{2}\right)=c\left(\sigma\|u\|_{L^{2}(\Omega)}^{2}+\|\nabla u\|_{L^{2}(\Omega)}^{2}\right)
$$

where $c$, being the operator norm of $T$ with respect to $H^{1}(\Omega), H^{1 / 2}(\Gamma)$, is independent of $\sigma$.

(b) Let us define the extension operator $P_{\sigma}: H^{1 / 2}(\Gamma) \rightarrow H^{1}(\Omega)$ by $P_{\sigma} u(x)=$ $(P \tilde{u})\left(\sigma^{1 / 2} x\right)$, where $P$ is a continuous extension operator in the case $\sigma=1$. The estimate of the operator norm of $P_{\sigma}$ with respect to the norms $\|\cdot\|_{\sigma, 1, \Omega},\|\cdot\|_{\sigma, 1 / 2, \Gamma}$ is analogous to the estimate of $T$ :

$$
\begin{aligned}
\left\|P_{\sigma} u\right\|_{\sigma, 1, \Omega}^{2} & \sim \sigma\left\|P_{\sigma} u\right\|_{L^{2}(\Omega)}^{2}+\left\|\nabla P_{\sigma} u\right\|_{L^{2}(\Omega)}^{2} \\
& =\sigma^{\frac{2-n}{2}}\left(\|P \tilde{u}\|_{L^{2}(\Omega)}^{2}+\|\nabla P \tilde{u}\|_{L^{2}(\Omega)}^{2}\right) \\
& \leq c \sigma^{\frac{2-n}{2}}\left(\|\tilde{u}\|_{L^{2}(\Gamma)}^{2}+\left\|\left(-\Delta_{\Gamma}\right)^{1 / 4} \tilde{u}\right\|_{L^{2}(\Gamma)}^{2}\right) \\
& =c\left(\sigma^{\frac{1}{2}}\|u\|_{L^{2}(\Gamma)}^{2}+\left\|\left(-\Delta_{\Gamma}\right)^{1 / 4} u\right\|_{L^{2}(\Gamma)}^{2}\right)
\end{aligned}
$$

where $c$, the norm of $P$, is independent of $\sigma$.

Part 2: Generalization to the case where $\Omega_{i}=\left(0, a_{1}\right) \times \cdots \times\left(0, a_{n}\right) \subset \mathbb{R}^{n}$ is a cuboid and $\Gamma_{i}=\{0\} \times\left(0, a_{2}\right) \times \cdots \times\left(0, a_{n}\right)$ is one face of $\Omega_{i}$ and $\Gamma_{i-1}=$ $\left\{a_{1}\right\} \times\left(0, a_{2}\right) \times \cdots \times\left(0, a_{n}\right)$ is another face: 
(a) The continuity of the trace operator $T: H_{i, \partial \Omega}^{1} \rightarrow H_{00}^{1 / 2}\left(\Gamma_{i}\right)$ with respect to the norms $\|\cdot\|_{\sigma, 1, \Omega_{i}},\|\cdot\|_{\sigma, 1 / 2, \Gamma_{i}}$, uniform with respect to $\sigma$, is obtained as follows:

Let us assume $\sigma \geq 1$ at first. It is sufficient to consider $T$ restricted to the space $\left\{u \in H^{1}\left(\Omega_{i}\right)|u|_{\partial \Omega_{i} \backslash \Gamma_{i}}=0\right\} \subset H_{i, \partial \Omega}^{1}$ : If $u \in H_{i, \partial \Omega_{i}}^{1}$ restricted to $\Gamma_{i-1}$ is different from zero, we can define $u^{*}\left(x_{1}, \ldots, x_{n}\right)=\left(1-x_{1} / a_{1}\right) u\left(x_{1}, \ldots, x_{n}\right)$ having the same trace on $\Gamma_{i}$ as $u$. Since $\sigma \geq 1$, one can easily estimate $\left\|u^{*}\right\|_{\sigma, 1, \Omega_{i}} \leq c\|u\|_{\sigma, 1, \Omega_{i}}$ with a $c>0$ independent of $\sigma$. It remains to estimate $T u=T u^{*}$ by $u^{*}$.

Hence, let $u \in H^{1}\left(\Omega_{i}\right)$ be given with $\left.u\right|_{\partial \Omega_{i} \backslash \Gamma_{i}}=0$. We can extend $u$ by zero to a function defined on the half space $\left\{x_{1}>0\right\} \times \mathbb{R}^{n-1}$. Let us denote this extension again by $u$. We apply the trace operator $T$ from Part 1 of the proof to the extended $u ; T u \in H^{1 / 2}\left(\mathbb{R}^{n-1}\right)$ has compact support in $\Gamma_{i}$, then. For the estimation of the restriction $R T u \in H^{1 / 2}\left(\Gamma_{i}\right)$ of $T u$, we use the norm equivalence

$$
\|v\|_{H_{00}^{1 / 2}\left(\Gamma_{i}\right)} \sim\|v\|_{H^{1 / 2}\left(\mathbb{R}^{n-1}\right)} \quad \forall v \in H^{1 / 2}\left(\mathbb{R}^{n-1}\right),\left.v\right|_{\mathbb{R}^{n-1} \backslash \Gamma_{i}}=0,
$$

which can be deduced from the norm representation

$$
\|v\|_{H_{00}^{1 / 2}\left(\Gamma_{i}\right)}^{2} \sim\|v\|_{H^{1 / 2}\left(\Gamma_{i}\right)}^{2}+\int_{\Gamma_{i}} \frac{|v(x)|^{2}}{\operatorname{dist}\left(x, \partial \Gamma_{i}\right)} d x
$$

(14, Chap. 1 (11.53)]). From the norm equivalence we obtain

$$
\|R T u\|_{H_{00}^{1 / 2}\left(\Gamma_{i}\right)}^{2} \leq c\|T u\|_{H^{1 / 2}\left(\mathbb{R}^{n-1}\right)}^{2} .
$$

Together with the trivial equality

$$
\sigma^{\frac{1}{2}}\|R T u\|_{L^{2}\left(\Gamma_{i}\right)}^{2}=\sigma^{\frac{1}{2}}\|T u\|_{L^{2}\left(\mathbb{R}^{n-1}\right)}^{2},
$$

the estimate

$$
\begin{aligned}
& \|R T u\|_{\sigma, \frac{1}{2}, \Gamma_{i}}^{2} \leq c\|T u\|_{H^{1 / 2}\left(\mathbb{R}^{n-1}\right)}^{2}+\sigma^{\frac{1}{2}}\|T u\|_{L^{2}\left(\mathbb{R}^{n-1}\right)}^{2} \\
& \quad=\quad c\left\|\left(-\Delta_{\mathbb{R}^{n-1}}\right)^{1 / 4} T u\right\|_{L^{2}\left(\mathbb{R}^{n-1}\right)}^{2}+\left(c+\sigma^{\frac{1}{2}}\right)\|T u\|_{L^{2}\left(\mathbb{R}^{n-1}\right)}^{2} \\
& \quad \leq \max \left\{c, c \sigma^{-1 / 2}+1\right\}\|T u\|_{\sigma, \frac{1}{2}, \mathbb{R}^{n-1}} \leq c\|T u\|_{\sigma, \frac{1}{2}, \mathbb{R}^{n-1}}
\end{aligned}
$$

follows with $c$ independent of $\sigma$, where the last step is valid for $\sigma \geq 1$ and $c$ is generic. $\|T u\|_{\sigma, \frac{1}{2}, \mathbb{R}^{n-1}} \leq c\|u\|_{\sigma, 1, \Omega_{i}}, c$ independent of $\sigma$, follows from Part 1 of the proof. Altogether, $\|R T u\|_{\sigma, \frac{1}{2}, \Gamma_{i}} \leq c\|u\|_{\sigma, 1, \Omega_{i}}$ follows.

The case $\sigma<1$ is handled as follows: For $u \in H_{i, \partial \Omega}^{1}$,

$$
\|T u\|_{\sigma, \frac{1}{2}, \Gamma_{i}} \leq\|T u\|_{H_{00}^{1 / 2}\left(\Gamma_{i}\right)} \leq c\|u\|_{H^{1}\left(\Omega_{i}\right)} \leq c\|\nabla u\|_{L^{2}\left(\Omega_{i}\right)} \leq c\|u\|_{\sigma, 1, \Omega_{i}},
$$

where the ordinary trace theorem $(\sigma=1)$ was used in the second step and a Poincaré inequality was used in the third step.

The generalization of part (b) of the lemma from the half-space situation to the cuboid geometry is obtained as follows:

Let $O$ be an open neighborhood of $\Gamma_{i}$ and let $\Phi \in W^{1, \infty}(O)$ be a Lipschitzcontinuous bijective mapping of $O$ to a bounded subset $M \subset \mathbb{R}^{n}$ such that $O \cap \partial \Omega_{i}$ is mapped to $M \cap\left\{x_{1}=0\right\} \times \mathbb{R}^{n-1}, O \cap \Omega_{i}$ is mapped to $M \cap\left\{x_{1}>0\right\} \times \mathbb{R}^{n-1}$, and such that this mapping, restricted to $\Gamma_{i}$, is the identity. Let $\psi$ be a smooth function with compact support in $O$ and $0 \leq \psi \leq 1$ on $O$ and $\psi=1$ on $\Gamma_{i}$. We will construct an extension operator $P_{\sigma, i}: H_{00}^{1 / 2}\left(\Gamma_{i}\right) \rightarrow H^{1}(\Omega),\left.\left(P_{\sigma, i} u\right)\right|_{\partial \Omega_{i} \backslash \Gamma_{i}}=0$, with norm bounded uniform with respect to $\sigma$ : 
A given function $u \in H_{00}^{1 / 2}\left(\Gamma_{i}\right)$ can be continuously extended by zero to a function in $\left\{x_{1}=0\right\} \times \mathbb{R}^{n-1}$ (see norm equivalences in part (a). The extension is again denoted by $u$. Then, the extension operator $P_{\sigma}$ from Part 1 of the proof can be applied. We define the extension operator $P_{\sigma, i}$ by $\left(P_{\sigma, i} u\right)(x)=\psi(x) P_{\sigma} u(\Phi(x))$, $x \in O \cap \Omega_{i}$, for all $\sigma \geq \sigma_{0}=\max _{x \in O}|\nabla \psi|^{2}, P_{\sigma}$ from Part 1. Obviously, the support of $P_{\sigma, i} u$ is contained in $O \cap \overline{\Omega_{i}},\left.\left(P_{\sigma, i} u\right)\right|_{\Gamma_{i}}=u$ and $\left.\left(P_{\sigma, i} u\right)\right|_{\partial \Omega_{i} \backslash \Gamma_{i}}=0$. We have to check the continuity of $P_{\sigma, i}$, uniform with respect to $\sigma$ :

$$
\begin{aligned}
& \left\|P_{\sigma, i} u\right\|_{\sigma, 1, \Omega_{i}}^{2}=\sigma\left\|\psi\left(P_{\sigma} u\right) \circ \Phi\right\|_{L^{2}\left(O \cap \Omega_{i}\right)}^{2}+\left\|\nabla\left(\psi\left(P_{\sigma} u\right) \circ \Phi\right)\right\|_{L^{2}\left(O \cap \Omega_{i}\right)}^{2} \\
& \quad \leq \quad \sigma\left\|\left(P_{\sigma} u\right) \circ \Phi\right\|_{L^{2}\left(O \cap \Omega_{i}\right)}^{2}+2\left\|\left(P_{\sigma} u\right) \circ \Phi \nabla \psi\right\|_{L^{2}\left(O \cap \Omega_{i}\right)}^{2}+2\left\|\psi \nabla\left(P_{\sigma} u \circ \Phi\right)\right\|_{L^{2}\left(O \cap \Omega_{i}\right)}^{2} .
\end{aligned}
$$

Since $|\psi| \leq 1,|\nabla \psi| \leq \sqrt{\sigma_{0}} \leq \sqrt{\sigma}$ on $O$, we obtain

$$
\begin{aligned}
\left\|P_{\sigma, i} u\right\|_{\sigma, 1, \Omega_{i}}^{2} & \leq 3 \sigma\left\|\left(P_{\sigma} u\right) \circ \Phi\right\|_{L^{2}\left(O \cap \Omega_{i}\right)}^{2}+2\left\|\nabla\left(\left(P_{\sigma} u\right) \circ \Phi\right)\right\|_{L^{2}\left(O \cap \Omega_{i}\right)}^{2} \\
& \leq c_{1}\left(\sigma\left\|P_{\sigma} u\right\|_{L^{2}(M \cap \Omega)}^{2}+\left\|\nabla\left(P_{\sigma} u\right)\right\|_{L^{2}(M \cap \Omega)}^{2}\right) \\
& \leq c_{1}\left(\sigma\left\|P_{\sigma} u\right\|_{L^{2}(\Omega)}^{2}+\left\|\nabla\left(P_{\sigma} u\right)\right\|_{L^{2}(\Omega)}^{2}\right) \\
& \leq c_{1} c_{2}\left(\sqrt{\sigma}\|u\|_{L^{2}\left(\Gamma_{i}\right)}^{2}+\left\|\left(-\Delta_{\Gamma_{i}}\right)^{1 / 4} u\right\|_{L^{2}\left(\Gamma_{i}\right)}^{2}\right)
\end{aligned}
$$

where $c_{1}$ depends on $\Phi$ and $c_{2}$ is the bound for $P_{\sigma}$ from Part 1 .

It remains to handle the case $\sigma<\sigma_{0}$. For all $\sigma<\sigma_{0}$ we define $P_{\sigma, i} u$ for $u \in$ $H_{00}^{1 / 2}\left(\Gamma_{i}\right)$ to be the ordinary extension operator $P: H_{00}^{1 / 2}\left(\partial \Omega_{i}\right) \rightarrow H^{1}(\Omega)$, applied to $u$ after extension by zero to $\partial \Omega_{i} \backslash \Gamma_{i}$. Using the continuity of $P$ and a Poincaré inequality in $H_{00}^{1 / 2}\left(\Gamma_{i}\right)$, we get

$$
\begin{aligned}
\left\|P_{\sigma, i} u\right\|_{\sigma, 1, \Omega_{i}}^{2} & =\|P u\|_{\sigma, 1, \Omega_{i}}^{2} \leq \max \{\sigma, 1\}\|P u\|_{H^{1}\left(\Omega_{i}\right)}^{2} \\
& \leq c \max \left\{\sigma_{0}, 1\right\}\|u\|_{H_{00}^{1 / 2}\left(\Gamma_{i}\right)}^{2} \leq c \max \left\{\sigma_{0}, 1\right\}\left\|\left(-\Delta_{\Gamma_{i}}\right)^{1 / 4} u\right\|_{L^{2}\left(\Gamma_{i}\right)}^{2} \\
& \leq c \max \left\{\sigma_{0}, 1\right\}\|u\|_{\sigma, 1 / 2, \Gamma_{i}}^{2}
\end{aligned}
$$

where $c$ is generic and independend of $\sigma$.

Part 3: The generalization to domains of more general shape works like the usual generalization of the well-known trace theorem by application of local maps.

\section{ACKNOWLEDGMENTS}

The author would like to thank W. Borchers and the reviewers of this article for their useful suggestions.

\section{REFERENCES}

1. S. Blazy, W. Borchers, U. Dralle, Parallelization Methods for a Characteristic's Pressure Correction Scheme, in: Flow simulation with high-performance computers II, Hirschel (ed.), Notes on Numerical Fluid Dynamics, Vol. 38, Braunschweig, Vieweg 1996. MR2000g:76001

2. W. Borchers, M.Y. Forestier, S. Kräutle, R. Pasquetti, R. Peyret, R. Rautmann, N. Roß, C. Sabbah, A Parallel Hybrid Highly Accurate Elliptic Solver for Viscous Flow Problems, Numerical Flow Simulation I, Notes on Num. Fluid Mech. Vol. 66, Hirschel (ed.), pp. 3-24, Springer Verlag 1998. MR99i:76104

3. W. Borchers, S. Kräutle, R. Pasquetti, R. Peyret, R. Rautmann, Multi-domain Finite Element-Spectral Chebyshev Parallel Navier-Stokes Solver for Viscous Flow Problems, Numerical Flow Simulation III, Notes on Num. Fluid Mech. Vol. 82, Hirschel (ed.), pp. 3-17, Springer Verlag 2003.

4. J. Bramble, J. Pasciak, J. Xu, Parallel multilevel preconditioners, Math. Comp., 55 (1990), pp. 1-22. MR1023042 (90k:65170) 
5. S.C. Brenner, The condition number of the Schur complement in domain decomposition, Numer. Math. 83 (1999), pp. 187-203. MR2000g:65114

6. S.C. Brenner, Lower bounds for nonoverlapping domain decomposition preconditioners in two dimensions, Math. Comp. 69 (2000), pp. 1319-1339. MR2001a:65156

7. C. Canuto, M.Y. Hussaini, A. Quarteroni, T.A. Zang, Spectral Methods in Fluid Dynamics, Springer Verlag, New York 1988. MR89m:76004

8. C. Farhat, F.-X. Roux, A method of finite element tearing and interconnecting and its parallel solution algorithm, Int. J. Num. Methods Engin. 32, pp. 1205-1227 (1991).

9. C. Farhat, J. Mandel, F.-X. Roux, Optimal convergence properties of the FETI domain decomposition method, Comput. Methods Appl. Mech. Engrg. 115 (1994), pp. 365-385. MR95d:65091

10. C. Farhat, L. Crivelli, F.-X. Roux, A transient FETI methodology for large-scale parallel implicit computations in structural mechanics, Int. J. Numer. Methods Eng. 37 (1994), pp. 1945-1975.

11. W. Guo, L.S. Hou, Generalizations and accelerations of Lions' nonoverlapping domain decomposition method for linear elliptic PDE, SIAM J. Numer. Anal. 41 (2003), pp. 2056-2080.

12. A. Klawonn, O. Widlund, FETI and Neumann-Neumann iterative substructuring methods: Connections and new results, Comm. Pure Appl. Math. 54 (2001), pp. 57-90. MR2001i:65131

13. S. Kräutle, A Navier-Stokes solver based on CGBI and the method of characteristics, Doctoral thesis, Erlangen, 2001. http://www.am.uni-erlangen.de/am1/publications/dipl_phd_thesis/ PhD_Kraeutle.ps.gz.

14. J.L. Lions, E. Magenes, Non-Homogeneous Boundary Value Problems and Applications I, Springer Verlag, Berlin-Heidelberg-New York 1982. MR50:2670

15. J.L. Lions, O. Pironneau, Non-overlapping domain decomposition for evolution operators, C. R. Acad. Sci. Paris, t. 330, Série I (2000), pp. 1-8. MF2001f:65110b

16. P.L. Lions, On the Schwarz alternating method III: A variant for nonoverlapping subdomains, Proceedings of the Third International Symposium on Domain Decomposition Methods for Partial Differential Equations, Chan, Glowinski, Périaux, Widlund (eds.), Philadelphia, PA, 1990, SIAM, pp. 202-223. MR91g:65226

17. J. Mandel, R. Tezaur, Convergence of a substructuring method with Lagrange multipliers, Num. Math. 73 (1996), pp. 473-487. MR97h:65142

18. J. Mandel, R. Tezaur, On the convergence of a dual-primal substructuring method, Num. Math. 88 (2001), pp. 543-558. MR2002b:65161

19. H. Triebel, Interpolation Theory, Function Spaces, Differential Operators, North-Holland Publishing Company, Amsterdam, New York, Oxford, 1978. MR80i:46032b

Institut für Angewandte Mathematik, Universität Erlangen-NÜrnberG, MartensStrasse 3, 91054 Erlangen, Germany

E-mail address: kraeutle@am.uni-erlangen.de 